\title{
Near-Earth Space Radiation Models
}

\author{
Michael A. Xapsos, Senior Member, IEEE, Patrick M. O’Neill and T. Paul O’Brien
}

\begin{abstract}
Review of models of the near-Earth space radiation environment is presented, including recent developments in trapped proton and electron, galactic cosmic ray and solar particle event models geared toward spacecraft electronics applications.
\end{abstract}

Index Terms - galactic cosmic rays, solar particle events, space radiation models, trapped particles

\section{INTRODUCTION}

$\mathrm{T}$ here are a number of environmental hazards that spacecraft must be designed for, which includes low energy plasma, particle radiation, neutral gas particles, ultraviolet and $\mathrm{x}$-ray radiation, micrometeoroids and orbital debris. This review is focused on hazards present for devices and integrated circuits in the near-Earth orbital radiation environment. Hence it is mainly concerned with three categories of particle radiations in space. The first is particles trapped by the Earth's magnetic field, also known as the Van Allen Belts. The second is the comparatively low-level flux of highly energetic ions that originate outside of our solar system called galactic cosmic rays. The third is bursts of radiation emitted by the sun, characterized by high fluxes of protons and heavy ions, referred to as solar particle events.

In order to have reliable, cost-effective designs and implement new space technologies, the climatology of the radiation environment must be understood and accurately modeled to eliminate or make manageable residual risk due to total ionizing dose (TID), displacement damage (DD), single event effects (SEE) and electrostatic discharge (ESD). Underestimating radiation levels leads to excessive risk and can result in degraded system performance and loss of mission lifetime. Overestimating radiation levels can lead to excessive shielding, reduced payloads, reduced payload performance, over-design and increased cost.

A summary of the types of radiation that are generally a concern for the electronic systems and instrumentation in Earth orbiting spacecraft and some of their properties is presented in

Manuscript received July 12, 2012; revised October 4, 2012.

M. A. Xapsos is with the National Aeronautics and Space Administration, Goddard Space Flight Center, Greenbelt, MD 20771 USA (phone: 301-2862263; fax: 301-286-4699; e-mail: Michael.A.Xapsos@nasa.gov).

P. M. O'Neill is with the National Aeronautics and Space Administration, Johnson Space Center, Houston, TX 77058 USA (e-mail: Patrick.M.ONeill@nasa.gov).

T. P. O'Brien is with The Aerospace Corporation, Chantilly, VA 20151 USA (e-mail: Paul.OBrien@aero.org).
Table I. Also shown are the radiation effects they primarily cause and general effectiveness of shielding in mitigating these effects.

There are several factors that are driving the development of improved space radiation models. Foremost is the pervasive use of high performance commercial-off-the-shelf (COTS) microelectronics to achieve increased system performance at lower cost but often at higher radiation risk, making improvement of radiation models critical. New space data are continually becoming available from modern instrumentation allowing models to be updated and further developed. Techniques that have been used previously in other fields are being adapted for these space applications.

There are a number of excellent publications on the space radiation environment in the form of review papers [1]-[3] and short course manuscripts [4]-[8]. In this paper we present an updated review.

\section{Models of Trapped Radiation AT EARTH}

\section{A. Background}

The magnetically trapped radiation at Earth is known as the Van Allen belts. It consists of energetic electrons from 100 $\mathrm{keV}$ to $10 \mathrm{~s}$ of $\mathrm{MeV}$ and protons from $\sim 100 \mathrm{keV}$ up to around a $\mathrm{GeV}$. The belts are organized into an inner zone and an outer zone separated by a slot region. Below $100 \mathrm{keV}$, a plasma population, known as the ring current, is also magnetically confined in this region. These particle populations together cause TID, DD, SEE, and vehicle charging, which leads to ESD. For particles below about $100 \mathrm{MeV}$, solar wind driven geomagnetic activity leads to dynamic variations of orders of magnitude in particle flux. It is believed that above about 100 $\mathrm{MeV}$, the particle population (protons) is comparatively stable, responding mainly to solar cycle variations in the cosmic ray source and atmospheric loss. For the purposes of satellite design, the physical processes of the belts are of far less concern than the statistical climatology. At present, physics based models of the radiation belts are not utilized for satellite design, although we will discuss how that is beginning to change.

Climatology models of the Earth's natural trapped radiation environment are undergoing revolutionary change in response to new requirements from the satellite industry, new data sources, and new modeling techniques. It has been clear for 
many years [9], [10] that the standard National Aeronautics and Space Administration (NASA) models Aerospace, Electrons, version 8 (AE8) [11] and Aerospace, Protons, version 8 (AP8) [12] are inadequate. They do not cover the full energy range needed; do not represent dynamics (space weather); and do not provide indications of uncertainty that would be needed to establish risk-based margins.

Toward the middle of the last decade, several outreach efforts were undertaken by NASA and the Space Technology Alliance [e.g., 13] to determine the requirements for new models of the Earth's trapped radiation environment. As a result of these efforts, the scientific community began research efforts to meet the new requirements [e.g., 14]. Table II summarizes the new requirements, adopted for AE9/AP9. Additionally, the new models need improved accuracy, temporal dynamics, and indications of uncertainty. Most importantly, to truly replace the AE8/AP8 de-facto standards, the new models would need to cover the entire energy and spatial domain covered by AE8/AP8.

In the remainder of section II, we will review different categories of models, provide representative examples, and conclude with a discussion of outstanding challenges.

\section{B. Ad hoc models}

The most straightforward way to build a trapped radiation model is to organize data from a single mission or orbit. Such an ad hoc model often covers a limited region of space. This approach can lead to confusion when different models overlap and disagree [15], however it is a valuable stop-gap measure between updates to global models.

There are two broad classes of ad hoc models: average environments that capture dynamics on solar cycle timescales and worst case environments that capture short-term geomagnetic storm dynamics in specific orbits. The former are developed mainly to address total dose issues, the latter for internal charging and single event effects.

For low Earth orbit, data from National Oceanic and Atmospheric Administration/Television Infrared Observation Satellite/Polar Operational Environmental Satellite (NOAA/TIROS/POES) [16], [17] and Solar, Anomalous and Magnetospheric Particle Explorer (SAMPEX) [18] sensors have been combined into a series of proton environment models. For medium-to-high altitude orbits, the Combined Release and Radiation Effects Satellite (CRRES) models provide proton [19] and electron [20] environments. The CRRES electron model includes a worst day state and a series of states for different levels of magnetic activity on monthly timescales. This is an attempt to model belt dynamics from a geophysical perspective. While the CRRES models cover a large part of the domain provided by AE8/AP8, they do not cover the entire domain or energy range of the older models. It should be noted that Vampola [21] provided an update to the solar minimum state of AE8 based on CRRES data, and some have used this in place of the older solar minimum state, e.g., [22].

More recently, electron models have been developed specifically for geostationary Earth orbit (GEO) using data from Los Alamos National Laboratory (LANL) sensors and the Data Relay Test Satellite (DRTS) [23]. The latest version of these international geostationary electron (IGE) models extends the electron energy range down to the plasma regime and provides three severity levels. In preparation for the Galileo global navigation satellite system (GNSS), an ad hoc electron model was developed using data mainly from Global Positioning System (GPS) satellites [24].

The ad hoc models described so far have been similar to AE8/AP8 in that they are models of the average or typical radiation environment. Some include indications of uncertainty, but those "error bars" are not intended to describe short term dynamics from which one might determine a worst case environment, for example, for internal charging. Generating worst case spectra is somewhat more challenging. Reference [25] provided worst case electron spectra for several orbits using the worst day observed by CRRES. Reference [26] used extreme value statistics to produce worst case electron spectra for geostationary and 12-hour Molniya orbits.

As one would expect, the ad hoc models have been adopted piecemeal throughout the community. A more comprehensive approach has long been desired. Two methods promise to extend the advances of the ad hoc models to the global environment: reanalysis climatology and Monte Carlo models.

\section{Reanalysis Climatology}

Reanalysis represents a potentially powerful new tool for specifying the global radiation environment. Reanalysis refers to the use of data assimilation for reconstructing the long-term state of a system. In data assimilation, typically a physicsbased model is run to evolve the state forward in time, and then an assimilation step is used to adjust the state to better match concurrent data. Such models can seamlessly and simultaneously incorporate data from numerous missions, thus resolving one of the fundamental concerns about ad hoc models.

For the radiation belts, there are several data assimilative models [27]-[29], and one of them, Salammbo has been run for an entire solar cycle for electrons [30] and protons [31]. The Salammbo team has used the results of their solar-cycle length reanalysis to generate a global model of the electron belts [32]. So far, even though the underlying reanalysis evolves the state on timescales shorter than individual storms, only an average specification model has been developed, with separate yearly averages to capture intra- solar-cycle variability. However, with data tables representing the global state on shorter timescales, a user could reconstruct a flux time series at the vehicle for the whole mission duration (or at least the duration of the reanalysis) and compute any derived quantity desired. This would enable, for example, calculation of worst cases from a realistically varying environment.

\section{Monte Carlo models (AE9/AP9)}

A different approach that does not replicate actual events 
but which does represent temporal dynamics in a statistical manner is the Monte Carlo model, the basis of the AE9/AP9 models [33]. In a Monte Carlo model, a state vector is evolved forward in time using one of several techniques for generating surrogate time series data. In the AE9/AP9 models, the state vector is evolved using a multivariate, multi-lag low-order linear autoregressive model. That is, a model that computes the next state vector as a linear combination of previous state vectors and a random innovation term [34], [35]. Each member of the sequence of state vectors can be converted to a global flux map and projected onto the orbit of the vehicle under study.

By running the Monte Carlo simulation from many initial states and many realizations of the random innovation sequence, one can obtain a set of realistic scenarios of flux at the spacecraft for the entire mission duration. If one then computes the desired specification quantities from each scenario, one can perform statistical analysis across scenarios to obtain confidence intervals.

Developing Monte Carlo models requires knowledge of spatial and spatiotemporal correlations as well as statistical variations at individual spatial grid points. Such knowledge can be obtained by combining in situ data from multiple missions. Given the limitations of in situ data, such models rely heavily on interpolation and extrapolation. They could benefit substantially from correlations and flux statistics extracted from a reanalysis, which incorporates both physics knowledge and in situ data. Thus far reanalysis and Monte Carlo models have not been combined in this way.

\section{E. Examples}

We now present two examples of the use of trapped radiation models. First, Fig. 1 shows results of four models for the electron fluence at a GPS orbit. We include AE8 Min/Max [11], the GNSS model [24], a reanalysis [32] and AE9 [33], for a 10 year mission. AE8 Min and Max do not really represent error bars, since one is usually advised to add them together in some combination based on how much time the mission is expected to experience solar minimum and solar maximum conditions. Nonetheless, including both provides some idea of the uncertainty in the environment. The more recent GNSS model accomplishes this by providing lower, mean, and upper bounds, and it explicitly computes the solar cycle phase exposure using a launch year and duration as input. Finally, AE9 provides a number of Monte Carlo scenarios (40 in this case), from which a $50^{\text {th }}$ and $95^{\text {th }}$ percent confidence are computed.

Next we show in Fig. 2 an example of several internal charging environments at geostationary orbit: worst case 24hour averaged flux. We include AE8 Max times 30 (an arbitrary factor loosely based on observations), a CRRESderived spectrum from [25], a spectrum derived from extreme value analysis of LANL-GEO data by [26], and AE9 [33]. Again, AE9 provides a set of Monte Carlo scenarios from which percentiles can be computed. We should note that obtaining the AE9 worst case specification required simulating the entire 10 year mission 40 times, and computing running 24-hour averages at every time step, and accumulating the worst case for each scenario. For some orbits that move quickly through the radiation belts, such as low Earth orbit (LEO), time steps as small as 10 seconds must be taken, making the model run times very long. This is but one of the challenges facing developers of trapped radiation specifications.

\section{F. Challenges}

Several challenges remain, the largest of which is simply insufficient in situ data. NASA's Radiation Belt Storm Probes offers to make the best ever measurements of many parameters of the radiation belts, and promises to resolve some of the background issues suffered by sensors on CRRES (see, e.g., [19]). However, additional modeling capabilities also need to be developed.

The AE9/AP9 models include plasma specifications down to about $1 \mathrm{keV}$, but do not include local time dependence or a Monte Carlo capability for plasma dynamics. Without a Monte Carlo capability at plasma energies, there is no way to specify surface charging environments, which are a kind of worst case. It is not clear that an adequate Monte Carlo capability can ever be developed for plasmas, due to their short temporal and spatial scales. Also, the AP9 model does not address the systematic solar cycle variation of proton flux at low altitudes due to interactions with the thermosphere. Understanding variations on solar cycle timescales require long-term measurements, which exist in LEO, but with limited altitude and energy coverage.

Another challenge to both reanalysis climatology and Monte Carlo models is the integration of solar protons. Presently solar protons and trapped protons are treated separately. However, in reality they occur together, and any model attempting to capture dynamics must ultimately incorporate both.

Other lesser issues include: improving run-time for mission length simulations, especially if multiple scenarios are desired, understanding how to reasonably extrapolate the internal geomagnetic field decades into the future to account for motion of the South Atlantic Anomaly; and accounting for geomagnetic field variations that can appear, at a spacecraft, as dramatic, rapid changes in the radiation or plasma environment.

\section{GALACTIC COSMIC RAYS}

\section{A. Background}

This section emphasizes free space Galactic Cosmic Ray (GCR) measurements and model spectra - unshielded by materials and/or magnetic fields. All results are for "quiet times", i.e. times when there are no Forbush decreases (a GCR decrease following a solar event). Only GCR ions are considered (protons through uranium). The flux of galactic electrons, gamma rays and rare species are not considered important for either microelectronics or astronaut health. 


\section{B. Discovery}

In 1895 Henri Becquerel discovered ionizing radiation coming from natural ores. This led to searches for new radioactive substances, and it was soon found that radiation was omnipresent.

In 1912, the Austrian physicist, Victor Hess, took an electroscope on a hot air balloon flight. Hess discovered that the number of "rays" increased with altitude and he concluded that radiation must be entering the atmosphere from outer space. He received the Nobel Prize in 1936 for the discovery of cosmic rays.

\section{Origin}

GCRs are believed to be created by the exploding stars of our galaxy (the Milky Way) - especially core-collapse supernovae.

Stars are formed by the gravitational collapse of clouds of interstellar gas consisting mostly of hydrogen. The high density in the core of the new stars causes ignition of nuclear burning. A succession of nuclear reaction cycles converts protons into successive heavier elements. As shown in Fig. 3, the conversion of light elements into heavier ones is exothermic.

When all the light elements of a star are converted (burned), mainly into iron, heat is no longer produced because conversion of iron into heavier elements is endothermic. As the star cools, gravity causes its core to collapse. The implosion releases tremendous amounts of kinetic energy causing a core-collapse supernova. It is the shock waves, from this collapse, traveling out through the surrounding interstellar medium that accelerates cosmic rays.

Since the stellar winds that preceded the explosion contain only the elements up to iron, the GCR spectrum is mainly composed of elements from hydrogen up to the iron-nickel group and there is a sharp drop in abundance of elements heavier than iron.

\section{Composition}

Galactic cosmic rays (and solar too) are composed mainly of protons (hydrogen), about $90 \%$. There is also a significant amount of helium, about $9 \%$. The ions from lithium through the iron-nickel group (atomic number 28) account for most of the remaining $1 \%$. Elements above iron-nickel (up to atomic number of 92) are present, but they account for only $0.00003 \%$ of the GCRs [36].

\section{E. GCRs in the Heliosphere}

Fig. 4 demonstrates features of the energy spectrum of a typical GCR element - iron. The Local Interstellar Spectrum (LIS) is the spectrum that exists at the far edge of the heliosphere ( 100 AU). Enrico Fermi [37] of the University of Chicago Physics Department suggested that GCRs are accelerated in interstellar space by collisions with moving magnetic fields. This theory implies a power law in energy, which is in fact approximately observed for all GCRs at the higher energies (above $\sim 20 \mathrm{GeV} / \mathrm{n}$ ), where solar modulation does not affect the spectra.

The LIS is constant and isotropic at the heliosphere boundary. As these GCRs enter the region of the sun's magnetic field they are attenuated (modulated). So, the number of GCRs seen inside the heliosphere - especially at the lower energies $<20 \mathrm{GeV} / \mathrm{n}$ - is always smaller than in the LIS.

\section{F. Vintage GCR Measurements}

\section{1) Early}

Ionization chambers have been used to observe GCRs since the 1920s. Much about the nature of cosmic rays was discovered by these instruments - ground level events due to solar energetic particles, Forbush Decreases, and latitude and altitude dependence.

Throughout the 1930s balloon technology and cosmic ray detectors improved. By 1940, they reached 100,000 feet nearly the top of the atmosphere (TOA). By 1950 there were TOA balloon flights carrying radiation detectors capable of measuring cosmic ray energy spectra - mainly of hydrogen and helium - the most abundant elements.

Cosmic rays have been monitored continuously around the world since the early 1950s by the neutron monitor invented by John A. Simpson (University of Chicago) in 1948 [38]. Neutrons observed at ground level are mainly due to atmospheric interactions of high-energy GCR protons and helium and do not define the GCR energy spectrum. About once a year a large solar event produces a a "Ground Level Enhancement", (GLE) such as the one observed on February 23, 1956.

Fig. 5 shows that the GCR (neutron monitor) rates and sunspot number roughly anti-correlate. But, there are differences - the GCR peaks alternate between "plateau" and "pointed" every cycle - the sunspots do not exhibit this. Also, the GCRs tend to "lag" the sunspots. Shortly, we will see that it is not really the sunspots themselves that are causing the modulation but actually the Coronal Mass Ejections (CMEs). It just happens that the sunspot count is a good indicator of CME rate and can be used to determine the solar modulation level.

2) 1950 s - 1980s Balloon \& Satellite Energy Spectra

The 1950s - 1980s were the heyday for balloon and satellite observations. The detector technology: silicon barrier coincident hodoscopes, Cerenkov-scintillation telescopes, ionization spectrometers, photomultiplier tubes, and nuclear emulsions that were developed and perfected during these years.

From 1954 to 1964, the GCR spectrum measurements were made by ionization spectrometers and Cerenkov-scintillation telescopes aboard high altitude balloons. These flights were mainly at high magnetic latitudes in the northern US and Canada and only proton and helium spectra from $50 \mathrm{MeV} / \mathrm{n}$ to $50 \mathrm{GeV} / \mathrm{n}$ were measured - there were virtually no heavy ion observations. These instruments typically had collection areas of $100-600 \mathrm{~cm}^{2}-$ sr and floated $\sim 6$ hours at TOA.

In late 1963 the first satellite, the Interplanetary Monitoring Platform-1 (IMP-1), started measuring the proton and helium 
flux in the 50 - $100 \mathrm{MeV} / \mathrm{n}$ energy range with a $600 \mathrm{~cm}^{2}$ - sr collection area. For the first time, sufficient GCRs could be collected due to the large area of the telescope and longer collection time. These satellite measurements did not require atmosphere correction.

Pioneer 8, launched December 1967, in route to Jupiter, measured protons and helium with an advanced telescope. This single instrument covered the range from $6 \mathrm{MeV} / \mathrm{n}$ to $2 \mathrm{GeV} / \mathrm{n}$. One of the earliest observations was the now well known "anomolous component".

Anomalous Cosmic Rays (ACRs) [39] are limited to energies below $\sim 50 \mathrm{MeV} / \mathrm{n}$. ACRs may not be fully ionized and can therefore penetrate the magnetosphere more easily. However, the range of these ions is generally small and they are stopped by minimal shielding. For example, at $50 \mathrm{MeV} / \mathrm{n}$ the range of iron in aluminum is 40 mils, and the range of hydrogen is 420 mils.

\section{G. Modern GCR Measurements}

A number of GCR spectra have been measured in modern times (1990s and 2000s).

\section{1) Protons and Helium}

Protons and helium are so abundant their spectra are well defined by balloon and satellite instruments. The proton and helium observations by the Balloon-borne Experiment with Superconducting Spectrometer (BESS) from 1993 to 2002 and the Isotope Matter-Antimatter Experiment (IMAX) in 1992 improve the spectra for these light ions (Fig. 6).

BESS derives its precision from its large collection area and IMAX has a unique detector redundancy that improves its accuracy over the older measurements. IMAX provides the higher energy measurements - up to $200 \mathrm{GeV} / \mathrm{n}$.

The BESS instrument had a view area of $4200 \mathrm{~cm}^{2}$ - sr and measured for 17 hours. The IMAX instrument had a view area of only $142 \mathrm{~cm}^{2}$ - sr and measured for 16 hours. The BESS and IMAX data agree closely. The difference is due to solar modulation - the IMAX flight was slightly closer to Cycle 22 solar maximum so there was more modulation.

\section{2) Heavy Ions - Low Energy}

Although heavy ions comprise only $1 \%$ of the GCRs, their influence on electronics and humans is often more significant than that of the protons and helium. Almost $50 \%$ of the human dose equivalent comes from the $\mathrm{Z}>2$ ions [40].

The spectra for all of the ions from Lithium $(Z=3)$ to Nickel $(Z=28)$ are now very well defined by the NASA Advanced Composition Explorer (ACE) Cosmic Ray Isotope Spectrometer (CRIS) [40]. CRIS has continuously provided low energy ( $50-300 \mathrm{MeV} / \mathrm{n})$ spectra daily for these ions since 1997. That's all of solar cycle 23 and a good part of cycle 24! ACE is located at the Earth-Sun L1 libration point (about 1.5 million km from Earth) and the spacecraft principal investigators remove all of the "Forbush decreases". Clean, "quiet time" energy spectra are readily available on the ACE web site - http://www.srl.caltech.edu/ACE/. The ACE CRIS geometric factor is $250 \mathrm{~cm}^{2}-\mathrm{sr}$, much larger than most of the older satellite and balloon heavy ion measurements in the 50 -
$300 \mathrm{MeV} / \mathrm{n}$ range (Fig. 7).

3) Heavy Ions - High Energy

For higher energies, the NASA High Energy Astronomy Observatory-3 (HEAO-3) satellite carrying the French-Danish experiment -C2 [41] is the most precise data available today about the isotropic composition of GCRs above $2 \mathrm{GeV} / \mathrm{n}$.

The HEAO-3 -C2 collection factor was very large $(\sim 700$ $\mathrm{cm}^{2}$-sr) so even though the flux is low in this energy region excellent statistics are provided. Since the flux is constant at these high energies (not subject to solar modulation) meaningful data was continuously collected for 8 months. These measurements determine the LIS spectrum in this energy range. Fig. 7 shows the HEAO-3 -C2 measurements for 3 ions in comparison to the older balloon flights [42], [43]. The balloon flights were only one day long and their collection factors were about the same as HEAO-3 -C2's. Note the spread of the balloon data is larger than HEAO's.

The Alpha Magnetic Spectrometer-2 (AMS-2) is mounted on the International Space Station (ISS) and has collected GCR measurements since May 2011. The AMS-2 has a very large collection efficiency (4000 $\mathrm{cm}^{2}$-sr) and measures elements hydrogen through iron (http://www.ams02.org). The magnetic cutoff of ISS at its 57-degree inclination makes the AMS-2 high-energy spectra (up to several $\mathrm{TeV}$ ) very appealing.

For elements above iron few measurements exist and their relative GCR flux is derived from nuclear models of the creation process.

\section{H. GCR Models}

1) CRÈME86 - Adams' Original GCR Model

From 1981 to 1986 J.H. Adams, Jr. [44], [45] developed a very comprehensive model of the near-Earth ionizing radiation environment and its effect on microelectronics. The Cosmic Ray Effects in Microelectronics 1986 (CRÈME86) GCR model had a good foundation in the absolute measurements for hydrogen and helium flux from $10 \mathrm{MeV} / \mathrm{n}$ to $100 \mathrm{GeV} / \mathrm{n}$ using the data discussed above. However, for most of the elements CRÈME86 scaled the helium spectrum using a constant or energy dependent multiplier. Solar modulation was modeled as a sinusoidal variation with a 10.9-year period as the 22-year odd-even cycle was not yet known.

Adams' original work has been the foundation for all of the GCR models that have been developed since. Adams willingly provided FORTRAN source code for his original CRÈME86 model to spacecraft designers and health physicists and it was used extensively up to 1996 .

\section{2) CRÈME96}

In the early 1990s Adams organized a team to compile the most complete source of balloon and satellite GCR measurements possible. Adams' list showed $\sim 50$ papers on hydrogen \& helium data and about another 50 on heavier ions up to iron.

Adams also worked closely with the Naval Research Laboratory (NRL) team who developed CRÈME96. CRÈME96 used the Moscow State University model of 
Nymmik [46], which uses the International sun spot count (ISS\#), also called the Wolf number, to derive the solar modulation level. The GCR flux in Nymmik's model has an 815 month delay between sunspot and corresponding GCR levels - making future GCR flux predictable to about a year ahead.

CRÈME96 was recently improved to calculate GCR spectra for all of the cycles back to Cycle 1 ( 1745) based on accurate sunspot counts. The National Geographic Data Center (NGDC) is the best source for ISS\#.

The NRL CRÈME96 website no longer exists, but Vanderbilt's online program maintains the latest formulation, CRÈME-MC (https://crème.isde.vanderbilt.edu). CRÈME96 is often referenced in system design requirements. Predictions are within $\sim 20 \%$ of the actual quiet time GCR environment, which is more than adequate for system design. A paper by Tylka [47] gives an excellent description of CREME96.

3) Badhwar-O'Neill '10

Badhwar, as a member of Adams' team, researched each of Adams' early GCR references, adding some of his own. Badhwar used the data to determine the parameters for the spherically symmetric modulation model proposed by Fisk [48]. Badhwar's original model [49]-[51] used the Climax neutron monitor count as an indication of the current level of solar modulation.

The neutron monitor directly responds to the high-energy GCR proton flux. Therefore, the neutron monitor count directly measures the solar modulation level. The biggest source of error in using neutron count to determine solar modulation is that fluctuations in the Earth's atmosphere cause unaccountable fluctuations in the GCR flux to neutron ratio. Another shortcoming is that the GCR proton rigidity differs significantly from that of all the other GCR ions.

The modern Badhwar-O’Neill model (BO'10) uses sunspot count to determine the solar modulation level.

\section{Solar Modulation}

The heliosphere is very large and complex and much of its physics - such as co-rotating interaction regions, Forbush Decreases, outgoing coronal mass ejections and actual solar magnetic field - cannot be modeled by simple, empirical models.

Nymmik [46] has demonstrated that there are numerous advantages to deriving the modulation level from the mean International sunspot \#. He shows that care must be taken to account for time delays due to the response of the heliosphere to solar activity (sunspots), time delays due to the rigidity of the different GCR elements, and global effects due to the polarity of the heliospheric magnetic field - gradient and curvature drift.

Fig. 8 shows the ACE $80 \mathrm{MeV} / \mathrm{n}$ oxygen channel flux along with the BO'10 model. The model attempts to model the entire heliosphere with one modulation parameter (diffusion coefficient) when in fact, the modulation varies at different locations in the heliosphere. This modulation error affects both the shape of the GCR spectra and the overall magnitude of the spectrum. Each ion has its unique rigidity spectrum.

Therefore, the spectrum for an ion at different states of the heliosphere could be different - even if the modulation level is the same for each state.

Fig. 8 shows selected times that have the same solar modulation - but entirely different heliospheric conditions. The channel 1 flux near solar minimum at 23n-, 23n+, 24n+ all have the same modulation but they are of different cycles and opposite magnetic fields. Similarly, points $23 x-$ and $23 x+$ are near solar maximum. The oxygen flux-energy spectra for each of these times are shown in Fig. 9. The 3 spectra near solar minimum are within an average of $\sim 5 \%$ per point and the 2 solar maximum spectra are within $1 \%$. This shows that the overall solar modulation effect is essentially the same regardless of the state of the heliosphere. This helps justify a single, simple, empirical modulation parameter.

\section{J. Particle Transport in the Heliosphere}

The Fokker-Planck (F-P) equation accounts for cosmic ray propagation in the heliosphere due to diffusion, convection, and adiabatic deceleration. F-P assumes a steady state, spherically symmetric heliosphere. However there are two issues with this. The heliosphere changes dynamically and it is not spherical in shape.

The first issue - dynamics of the heliosphere - is easily incorporated in the models. The F-P model assumes a radially dependent diffusion coefficient whose magnitude depends on the solar modulation parameter as derived from sunspot count. The solar modulation parameter can be varied slowly with the solar cycle assuming that magnetic disturbances move away from the sun at the solar wind speed. These disturbances ${ }^{1}$ take about a year to reach the heliopause. The solar wind speed, $\sim 82$ AU/year $(400 \mathrm{~km} / \mathrm{s})$, is assumed constant. CREME96 treats this effect using an empirical time delay function between time of sunspot and time of flux.

The other issue, the lack of spherical symmetry of the heliosphere, is more difficult. Solar physicists have long known that the solar magnetic field changes sign at each solar maximum - a 22-year cycle. The solar magnetic field (B) points outward ( radially) in the northern solar hemisphere $(\mathrm{B}+)$ and inward in the southern hemisphere (B-) for 11 years after field reversal at solar maximum. This case is commonly referred to as positive solar field $\left(\mathrm{A}^{+}\right.$, or $\left.\mathrm{qA}>0\right)$ and this is the case shown in Fig. 10.

The solar magnetic cycle is out of phase with the solar activity cycle - it begins at solar maximum instead of solar minimum. Note the neutron monitor peaks in Fig. 5. The "plateau" GCR (neutron monitor) peaks are associated with A+ and that the "pointed" GCR peaks are associated with A-.

The gradient in the magnetic field near the poles combined with solar rotation allows positive ions to enter the heliosphere more easily from both of the solar poles (north and south) during positive solar magnetic field, [39,52]. In the A- case it's 
just the opposite, positive ions enter along the equatorial zone and they exit at both of the solar poles.

This polarity dependence is due to gradient and curvature drift and is not included in the F-P equation. However, the models account for this anti-symmetrical feature empirically. For example, the coefficients used in the models that derive the modulation parameter from the sunspot number differ by over $50 \%$ between A+ and A- magnetic cycles. Sunspots are roughly twice as effective in producing modulation during Acycles. There is more modulation for the GCRs entering along the current sheet (the equatorial zone) than those coming in along the poles.

\section{K. "Plateau" and "Pointed" Due to CMEs}

The drift effect is seen in the GCR observations. The A+ (Fig. 5) periods exhibit "plateaus" in the GCR flux. These "plateaus" are characterized by a rapid GCR rise followed by a delayed decline - hence the flat top.

Cliver and Ling [53] explain that this delay is because Coronal Mass Ejections (CMEs) are confined to the equator at the beginning of each solar cycle and move toward higher latitudes toward solar maximum. They argue that the primary modulation of GCRs is due to CMEs (not sunspots). Then, since the GCRs are mainly coming from the poles for the A+ case, their modulation is delayed until the CMEs move to higher latitudes. This delay is $\sim 1$ year.

Similarly, "pointed" GCR cycles occur during A- magnetic periods and these cycles respond immediately to the new solar cycle with minimal delay because the GCRs are entering along the equator where the CMEs are at the beginning of the cycle.

Note that sunspots start the cycle at high latitudes and move downward toward the equator during the cycle, but CMEs start the cycle at the equator and move toward the poles.

\section{Can GCRs Reach Deep Space by Way of Sun for A+?}

Drift may also have an effect on modulation of deep space GCRs. Fig. 11 shows the helium flux measured by Pioneer- 10 as it moved outward [54]. IMP-8 at 1 AU data is also shown. Note that the Pioneer flux is generally increasing (relative to the IMP-8 flux at $1 \mathrm{AU}$ ) as Pioneer moves closer to the heliopause.

The model closely matches the Pioneer flux prior to field reversal (A- to $\left.\mathrm{A}^{+}\right)$. However, after reversal, the model flux is significantly higher than Pioneer. We believe this error may be because the model does not account for the possibility that some of the GCR ions may have entered the heliosphere by way of the solar poles - as we expect it does for A+. Therefore, the isotropic flux of ions observed in the deep space location (Pioneer at 50-60 AU out) may have propagated past the solar poles and then outward along the neutral current sheet from the inner heliosphere. The model always assumes the ions are propagating inward from the outer parts of the heliopause rather than from the inside of the heliosphere.

\footnotetext{
${ }^{1}$ Disturbances don't come from sunspots. They come from outbursts due to the release of non-potential energy stored in the solar magnetic fields in active regions on the Sun.
}

\section{How Deep is the Deepest Minimum?}

One good reason to derive the solar modulation parameter from sunspots is that the International sun spot number is available for all 24 solar cycles - starting in 1750. Fig. 12 shows the variation in GCR flux for all 24 cycles of the sun. We tend to think that our last "deepest" minimum was outstanding. Indeed, it was deeper than the 1977 minimum that life sciences health physicists often use to represent the "typical minimum". However, the graph shows that there have been many "ups and downs" over the history and that the actual deepest minimum occurred back in 1810. The GCR flux at any of these various minima really doesn't vary more than $\sim 30 \%$. This may not be important for microelectronic system design, but it's very important when quality factors for human tissue are considered by the health physicist planning a deep space mission. These 24 minima would serve as a good foundation for determining a statistical "worst case" for GCRs.

\section{N. Single Event Effects and GCR Models}

Fig. 13 shows the GCR SEE error rate for typical microelectronic components calculated by the GCR models. Even though there are differences in the energy spectra used by each of the models - they differ by 10 - 20\% [51] - the calculated SEE error rate is practically identical (within 5\%) for a wide range of components. There are also differences in the calculated solar modulation even though each model derives the modulation from the sunspot number. Apparently, these differences "wash out" for the SEE calculation - at least at the 1977 solar minimum.

\section{O. Penetration}

GCRs are the most energetic and most penetrating particle radiation in the near-Earth environment. The annual GCR dose due to direct ionization is small, typically $\sim 10 \operatorname{rad}(\mathrm{Si})$ at solar minimum (Fig. 14). Most electronics can withstand this dose for years with no problem. However, humans are more sensitive - their lifetime limit is on the order of 25 rads. Shielding reduces the primary flux some, but also causes an increase in the secondary dose.

Although dose is not an issue for microelectronics, the SEE rates caused by the GCR spectrum can be. The steady GCR flux is the dominant cause of SEE for hard components in both low Earth orbit and deep space during quiet time conditions.

\section{P. GCR Model Improvements: What Does the Aerospace Community Need?}

The new, GCR spectra measurements made by BESS, IMAX, HEAO, ACE and AMS should be incorporated in the models.

The GCR models currently weigh the older data equally with the modern data. The old and new data should be properly weighted based on their collection efficiency.

The biggest source of error in the GCR models is the variation in the effect of solar modulation. Sunspots are a good indication and they work reasonably well, but additional indicators could supplement. 
Models should not use any low energy $(<2 \mathrm{GeV} / \mathrm{n})$ data that was collected and integrated over a long period. The solar modulation varies with time and this causes the spectrum to be distorted and causes the modulation to be uncorrelated with the data. The longest integration period for energies $<2 \mathrm{GeV} / \mathrm{n}$ should be about 3 months.

Very limited isotope measurements are available. The human dose for deep space travel and high latitude commercial aircraft traveling polar routes use the GCR models and they need better isotope resolution.

The curvature and drift effect needs to be incorporated in the GCR models. This should allow GCR spectra for heliospheric locations besides those at $1 \mathrm{AU}$ to be accurately accounted for. Currently, all models calculate GCR flux at 1 AU in the ecliptic plane and have no capability for higher latitudes or deeper space.

The GCR models do not have drift modeled accurately enough to calculate GCR flux at high heliospheric latitudes. Data are available such as that provided by Ulysses, which measured GCR spectra over both solar poles from 1994 to 2008.

\section{SOLAR PARTICLE EVENTS}

\section{A. Background}

It is believed that there are 2 categories of solar particle events and that each one accelerates particles in a distinct manner. Both phenomena are magnetic activity related. Solar flares result when the localized energy storage in the coronal magnetic field becomes too great and causes a burst of energy to be released. They tend to be electron rich, last for hours, and have unusually high ${ }^{3} \mathrm{He}$ content relative to ${ }^{4} \mathrm{He}$. A Coronal Mass Ejection (CME), on the other hand, is a large plasma eruption that drives a shock wave outward and accelerates particles. CMEs tend to be proton rich, last for days, and have small ${ }^{3} \mathrm{He}$ content relative to ${ }^{4} \mathrm{He}$. A review article by Reames gives a detailed account of the many observed differences between solar flares and CMEs [55].

CMEs are the type of solar particle event that is responsible for the major disturbances in interplanetary space and the major geomagnetic disturbances at Earth when they impact the magnetosphere. The total mass of ejected plasma in a CME is generally around $10^{15}$ to $10^{17}$ grams. Its speeds can vary from about 50 to $2500 \mathrm{~km} / \mathrm{s}$. It can take anywhere from about 12 hours to a few days to reach the Earth. Table I lists some further general characteristics.

All naturally occurring chemical elements ranging from protons to uranium are present in solar particle events. They can cause permanent damage such as TID and DD which is due mainly to the protons. The heavy ion content is a small percentage of the flux but nonetheless cannot be ignored. Heavy ions, as well as protons and alpha particles in solar particle events, can cause both transient and permanent SEE.

Fig. 15 illustrates the periodic yet stochastic nature of solar particle events. It is a plot of the daily $>0.88 \mathrm{MeV}$ solar proton fluences measured by the Interplanetary Monitoring Platform-8 (IMP-8) and Geostationary Operational Environment Satellites (GOES) over a 28 year period. The solar maximum and solar minimum time periods are shown in the Fig. to illustrate the dependence on solar cycle.

\section{B. Solar Proton Models}

The solar proton environment is a concern for all Earth orbiting spacecraft, especially those orbits having limited protection from the Earth's magnetic field such as polar and geostationary orbits. Solar proton models are generally more mature than solar heavy ion models because there are considerably more data available for protons. Section 1 discusses the probabilistic nature of events. Section 2 then describes the distribution of event magnitudes. Section 3 describes cumulative fluences over the course of a mission, and section 4 discusses worst-case events during a mission.

1) Probabilistic Nature of the Energy Release Process

Substantial efforts have been put into studies of the occurrence of solar particle events. One of the main goals is to find a reliable predictor of events. Despite this significant international effort, solar particle events can occur suddenly and without obvious warning. In addition to potential problems with electronic systems and instrumentation, this is a serious concern for manned space initiatives. Thus, there is strong motivation to develop predictive methods for solar particle events. It is hoped that the apparent stochastic character can be overcome and predictability achieved if precursor phenomena such as x-ray flares or magnetic topology signatures can be properly interpreted or if the underlying mechanisms are identified. Currently it is not possible to predict the time of occurrence and magnitude of solar particle events within narrow limits [56].

2) The Distribution of Solar Proton Event Magnitudes

Given that the occurrence of solar particle events is a stochastic phenomenon, it is important to accurately model the distribution of event magnitudes. However, in general it can be rather difficult to select a probability distribution for the situation where the data are limited. There have been a number of empirical assumptions that the event magnitudes can be represented by certain distributions. For example, power law distributions [57], [58], modified power law distributions [59] and lognormal distributions [60], [61] have been used, and each of these distributions has some favorable characteristics. However, it turns out that a truncated power law in the event fluence describes the distribution the most accurately, as shown in Fig. 16, which compares this result to $>30 \mathrm{MeV}$ solar proton event data during 21 solar maximum years [62]. This strong agreement indicates that this distribution captures the essential features of a solar proton event magnitude distribution. It is a power law for small event sizes and falls off rapidly for very large events.

\section{3) Cumulative Fluences}

During a space mission the solar particle event fluence that accumulates during the solar maximum time period is often the dominant contribution to the total fluence. Thus, much prior 
work focuses on this period of the solar cycle. A commonly used definition of the solar maximum period is the 7-year period that spans a starting point 2.5 years before and an ending point 4.5 years after a time defined by the maximum sunspot number in the cycle [63]. The remainder of the cycle is considered solar minimum.

Once the initial or underlying distribution of event sizes during solar maximum such as that shown in Fig. 16 is known, it can be used to determine the accumulated fluence for a period of time during solar maximum. Due to the stochastic nature of the events, confidence level approaches are often used so that risk-cost-performance tradeoffs can be evaluated by the spacecraft designer. The first such model was based on King's analysis of $>10$ to $>100 \mathrm{MeV}$ protons during solar cycle 20 [60], [64]. One "anomalously large" event, the wellknown August 1972 event, dominated the fluence of this cycle so the model has often been used to predict the number of such events expected for a given mission length at a specified confidence level. Using additional data, a model from JPL emerged in which Feynman et al. showed that the magnitude distribution of solar proton events during solar maximum is actually a continuous distribution between small events and the extremely large August 1972 event [61]. The JPL model uses Monte Carlo simulations to calculate the cumulative fluence during a mission at a given confidence level. An example of this is shown in Fig. 17 for $>30 \mathrm{MeV}$ protons. Thus, according to this model, there is approximately a $10 \%$ probability of exceeding a proton fluence of $10^{10} \mathrm{~cm}^{-2}$ for a 3year period during solar maximum. This corresponds to a $90 \%$ confidence level that this fluence will not be exceeded.

Other probabilistic models for cumulative fluence have been developed based on more recent and extensive data since the JPL91 model. These include a model from Moscow State University, which introduces solar cycle dependence by assuming that event numbers are directly proportional to sunspot numbers [65]. The NASA Emission of Solar Protons (ESP) model is based on Maximum Entropy and Bootstraplike statistical principles [66]. The European Space Agency (ESA) Virtual Timelines method invokes Levy and time dependent Poisson event distributions [67], although these time features could be disputed [68]. Comparisons of different models can be found in [3] and [67]. Readers may also wish to make their own comparisons using current web-based tools (http://www.spenvis.oma.be/; http://dev.sepem.oma.be/). A model for solar electrons has also been developed based on the statistical approach of the ESP model that is part of a new Interplanetary Electron Model [69].

\section{4) Worst Case Events}

An important consideration for spacecraft designers is the worst-case solar particle event that occurs during a mission. One approach is to design to a well-known large event such as that of October 1989 [70], or a hypothetical one such as a composite of the February 1956 and August 1972 events [71]. Energy spectra of some of the most severe solar proton events during solar cycles 19-22 are shown in Fig. 18 [72]. In addition, there are event classification schemes in which the magnitudes range from "small" to "extremely large" that may be helpful for design purposes [73], [74].

Additional information can be provided to the designer if a confidence level associated with the worst case event is known for a given mission length. The designer can then more systematically balance risk-cost-performance tradeoffs for the mission in a manner similar to what is done for cumulative fluences. Once the initial probability distribution such as that shown in Fig. 16 is determined it becomes possible to construct such a statistical model using extreme value theory.

In the usual central value statistics, the distribution for a random variable is characterized by its mean value and a dispersion indicator such as the standard deviation. Extreme value statistics, pioneered by Gumbel [75], focuses on the largest or smallest values taken on by the distribution. Thus, the "tails" of the distribution are the most significant. For this application the concern is with the largest values.

Examples of extreme value modeling of environmental phenomena such as floods, wave heights, earthquakes and wind speeds can be found in a number of places [75]-[77]. This modeling was first applied to radiation effects problems by Vail, Burke and Raymond in a study of high density memories [78]. It has turned out to be a useful tool for studying the response of large device arrays to radiation. Other radiation effects applications have been found for arrays of gate oxides [79], [80], sensor arrays [81], [82] and Erasable Programmable Read Only Memories (EPROMs) [83].

Fig. 19 shows probabilistic results determined by application of extreme value theory to the solar proton event distribution shown in Fig. 16 [62]. The ordinate represents the probability that the worst-case event encountered during a mission will exceed the $>30 \mathrm{MeV}$ proton fluence shown on the abscissa. Also shown in Fig. 19 by the vertical line denoted by "Design Limit" is the maximum event fluence parameter, which can be used as an upper limit guideline. This approach can be extended to include true solar cycle dependence [84]. Analogous results have been obtained for peak solar proton fluxes using this methodology [85] and other approaches [65], [67]. Peak flux is useful for analysis of worst case SEE rates.

An interesting question is whether the data base of solar particle events can be extended beyond space measurements. The so-called Carrington Event has been widely quoted by the radiation effects community as being a worst case magnitude event as determined from analysis of the 400-year nitrate record in polar ice cores [86]. However, the glaciology and atmospheric communities have disagreed with this interpretation of the nitrate record and it turns out the Carrington Event was not observed in most ice core records [87]. This casts doubt on worst case models directly developed based on the nitrate record.

In addition to the evaluation of solar proton event magnitude statistics it can also be helpful for the design engineer to know how much time during a mission that a given high intensity flux level is exceeded. This is true for both the total time and the longest continuous time period that level is 
exceeded. For example, estimates show that $>11 \mathrm{MeV}$ fluxes of $10^{4} \mathrm{p} /\left(\mathrm{cm}^{2}\right.$-s-sr $)$ are equaled or exceeded about 2 hours per year while fluxes of $10^{3} \mathrm{p} /\left(\mathrm{cm}^{2}\right.$-s-sr $)$ are equaled or exceeded about 1 day per year during solar maximum [88]. This type of analysis is a natural way to relate the solar particle environment directly to design goals, especially those of data requirements for space instrumentation.

\section{Solar Heavy Ion Models}

Solar heavy ion models are generally not as advanced as solar proton models due to the large number of heavy ion species and their relatively small fluxes compared to protons, which complicates measurements of elemental fluxes. For microelectronics applications, solar heavy ion models are needed primarily to assess SEE.

\section{1) Cumulative Fluences}

A recent advance in solar particle models has been the Prediction of Solar particle Yields for Characterization of Integrated Circuits (PSYCHIC) model [89], a fairly complete description of cumulative solar heavy ion fluences. This is an extension of the NASA ESP model [66]. Measured alpha particle energy spectra are scaled to proton energy spectra based on measurements from the IMP-8 and GOES instrumentation during the time period 1973 to 2001. The energy spectra of remaining major heavy elements - C, N, O, $\mathrm{Ne}, \mathrm{Mg}, \mathrm{Si}, \mathrm{S}$ and $\mathrm{Fe}$ - are scaled to the alpha particle energy spectra using measurements of the Solar Isotope Spectrometer (SIS) onboard the Advanced Composition Explorer (ACE) spacecraft over the most recent 7 year solar maximum period. This modern instrumentation provides excellent mass resolution during the high flux rates of events and has been a key in the development of this model. The remaining minor heavy elements are determined either from measurements made by the International Sun-Earth Explorer-3 (ISEE-3) spacecraft instrumentation or an abundance model based on current knowledge of solar photospheric abundances and processes. Results for integral fluence-energy spectra are shown in Fig. 20 for some of the major elements and a summed spectrum for atomic number $Z>28$.

Transformation of the energy spectra to LET spectra has been a useful parameterization for prediction of SEE rates in space. Insight to the LET spectra can be gained if they are examined in terms of elemental groupings as shown in Fig. 21. This is for the $50 \%$ confidence level, which is representative of long-term average solar particle event fluences. The total fluence for all elements, shown in this figure by the solid black line, is multiplied by a factor of 1.5 for clarity. It is composed of a proton curve $(Z=1)$ and an alpha particle curve $(Z=2)$. These are high fluence, low LET components where the fluence contribution drops sharply to zero at the so-called "Bragg Peak" or maximum LET. The contribution from $Z=3$ to 26 particles extends out to an LET of about $29 \mathrm{MeV}$ $\mathrm{cm}^{2} / \mathrm{mg}$ in silicon. The fluence contribution from this group is dominated by the 8 major elements mentioned above. For many SEE applications this group of elements has the greatest impact on SEE rates. Finally, the trans-iron group $(Z=27$ to
92) extends out to an LET of slightly over $100 \mathrm{MeV}-\mathrm{cm}^{2} / \mathrm{mg}$. Only limited measurements of these fluences exist and this portion of the spectrum relies to a large extent on the abundance model.

Due to the comparatively large fluences of solar protons compared to heavier solar energetic ions, protons are usually considered to be the dominant contribution to TID and DD effects. Figs. 20 and 21 show this quantitatively. In fact, the long-term solar particle dose deposited in silicon behind 100 mils of Al shielding shows that about $90 \%$ of the dose is deposited by protons, $9 \%$ by alpha particles, and $1 \%$ by heavier ions [89].

2) Worst Case Events

In an attempt to model worst-case events, the original CREME model [45] and subsequently the CRRES/SPACERAD Heavy Ion Model of the Environment (CHIME) [90] scaled heavy ion abundances to protons for individual events. This assumption that events with the highest proton fluxes should also be heavy ion rich led to worst-case event models that are quite conservative [91]. Modifications of the original CREME code were subsequently made in the Modeling and Analysis of Cosmic Ray Effects in Electronics (MACREE) model [92] to define a less conservative worstcase solar particle event. MACREE gives the option of using a model based on the measured proton and alpha particle spectra for the well-known October 1989 event and an abundance model that is 0.25 times the CREME abundances for atomic numbers, $\mathrm{Z}>2$. The updated CREME96 and CREME MC codes use the October 1989 event as a worst-case scenario. They provide three levels of solar particle intensity [70]. These are the "worst week", "worst day" and "peak flux" models, which are based on proton measurements from the GOES-6 and -7 satellites and heavy ion measurements from the University of Chicago Cosmic Ray Telescope on the IMP-8 satellite. The most extensive heavy ion measurements in the model are for $\mathrm{C}, \mathrm{O}$ and $\mathrm{Fe}$ ions [93]. It is noteworthy that the energy spectra of these 3 elements extend out to roughly 1 $\mathrm{GeV}$ per nucleon.

Comparisons to the CREME96 worst case models have been made with data taken by the Cosmic Radiation Environment Dosimetry (CREDO) Experiment onboard the Microelectronics and Photonics Test Bed (MPTB) between 2000 and 2002 [94]. The data show that 3 major events during this time period approximately equaled the "worst day" model. An example of this is shown in Fig. 22 for an event that occurred in November 2001.

\section{Challenges}

There are challenges that need to be met to allow improved protection of our space assets. A major long-term challenge for all space radiation models is to develop more physical and dynamic models of the environment. This is especially true for solar particle event models as the energy storage and release processes in the solar magnetic field are not understood in detail. Increased understanding of these processes would improve models and allow better anticipation of the 
occurrence and magnitude of events. Engineering models used during the spacecraft design phase are climatological models and have not been developed for operational usage. There is much interest in "nowcasting" models for operational usage for the purpose of astronaut protection [95]. Developing parallel models to be used in the operational regime would allow "nowcasting" solar particle event effects in electronic systems and instruments. This would support mission operations in making decisions on whether to operate through an event that is underway. It would also support the design phase of spacecraft by making available dynamic aspects of the solar particle environment to designers.

\section{SUMMARY}

A review of near-Earth space radiation models has been presented, including recent updates. The environment due to trapped protons and electrons, galactic cosmic rays and solar particle events poses challenges to mission designers in the form of total ionizing dose, displacement damage, single event effects and electrostatic discharges. The advancement of spacecraft and instrument design and their reliable operation depends on the continued development and improvement of these models.

\section{REFERENCES}

[1] J. Feynman and S.B. Gabriel, "High-energy charged particles in space at one astronomical unit”, IEEE Trans. Nucl. Sci., vol. 43, pp. 344-352, Apr. 1996.

[2] J.L. Barth, C.S. Dyer and E.G. Stassinopoulos, "Space, atmospheric, and terrestrial radiation environments”, IEEE Trans. Nucl. Sci., vol. 50, pp. 466-482, June 2003.

[3] S. Bourdarie and M.A. Xapsos, "The near-earth space radiation environment”, IEEE Trans. Nucl. Sci., vol. 55, pp. 1810-1832, Aug. 2008.

[4] J.L. Barth, "Modeling space radiation environments", in 1997 IEEE NSREC Short Course, Pascataway, NJ: IEEE Publishing, 1997.

[5] C.S. Dyer, "Space radiation environment dosimetry", in 1998 IEEE NSREC Short Course, Pascataway, NJ: IEEE Publishing, 1998.

[6] J. Mazur, "The radiation environment outside and inside a spacecraft", in 2002 IEEE NSREC Short Course, Pascataway, NJ: IEEE Publishing, 2002.

[7] M.A. Xapsos, "Modeling the space radiation environment”, in 2006 IEEE NSREC Short Course, Pascataway, NJ: IEEE Publishing, 2006.

[8] H. Garrett and I. Jun, "Spacecraft environment interactions" in 2011 IEEE NSREC Short Course, Pascataway, NJ: IEEE Publishing, 2011.

[9] E. J. Daly et al., "Problems with models of the radiation belts," IEEE Trans. Nucl. Sci., vol. 43, pp. 403-415, Apr. 1996.

[10] D. J. Rodgers, "Empirical radiation belt models," AGU Geophys. Monogr., American Geophysical Union, Washington, DC, 1997.

[11] J. Vette, "The AE-8 trapped electron model environment”, NSSDC 9124, NASA/GSFC, Greenbelt, MD, 1991.

[12] D. M. Sawyer and J. I. Vette, AP-8 trapped proton environment for solar maximum and solar minimum, NSSDC/WDC-A-R\&S 76-06, NASA/GSFC, Greenbelt, MD. 1976.

[13] J.M. Lauenstein, J. L. Barth, and D. G. Sibeck, "Toward the development of new standard radiation belt and space plasma models for spacecraft engineering," Space Weather, 3, S08B03, 2005

[14] G.P. Ginet and T.P. O’Brien "AE-9/AP-9 Trapped radiation and plasma models," TOR-2010(3905)-3, The Aerospace Corporation, El Segundo, CA, 2010.
[15] J. Vette, The NASA/National Space Science Data Center Trapped Radiation Environment Model Program (1964-1991), NSSD/WDC-AR\&S 91-29, NASA/GSFC, Greenbelt, MD, 1991.

[16] S.L. Huston, G.A. Kuck, and K.A. Pfitzer, "Low altitude trapped radiation model using TIROS/NOAA data", in Radiation Belts: Models and Standards, Geophys. Monogr. Ser., 97, J.F. Lemaire, D. Heynderickz, and D.N. Baker, Ed, pp. 119-124, AGU, Washington, D.C., 1996.

[17] S.L. Huston, Space Environment and Effects: Trapped Proton Model, NASA/CR-2002-211784, NASA Marshall Spaceflight Center, Huntsville, AL, 2002.

[18] D. Heynderickx, M. Kruglanski, V. Pierrard, J. Lemaire, M. D. Looper, and J. B. Blake, "A low altitude trapped proton model for solar minimum conditions based on SAMPEX/PET data," IEEE Trans. Nucl. Sci., vol. 46, pp. 1475-1480, Dec. 1999.

[19] M.S. Gussenhoven, E. G. Mullen, M. D. Violet, C. Hein, J. Bass, and D. Madden, "CRRES high energy proton flux maps," IEEE Trans. Nucl. Sci., vol. 40, pp. 1450-1457, Dec. 1993.

[20] D.H. Brautigam, M. S. Gussenhoven and E. G. Mullen, Quasi-Static Model of Outer Zone Electrons, IEEE Trans. Nucl. Sci., vol. 39, 17971803, Dec. 1992.

[21] A.L. Vampola "The ESA Outer Zone Electron Model Update, Environment Modelling for Space-based Applications," Symposium Proceedings (ESA SP-392), eds, W.Burke and T.-D. Guyenne, p.151, ESTEC Noordwijk, 18-20 September 1996.

[22] D. Heynderickx, B. Quaghebeur, J. Wera, E.J. Daly and H.D.R. Evans, "New radiation environment and effects models in the European Space Agency's Space Environment Information System (SPENVIS), Space Weather, 2, S10S03, 2004, doi:10.1029/2004SW000073.

[23] A. Sicard-Piet, S. A. Bourdarie, D. M. Boscher, R. H. W. Friedel, M. Thomsen, T. Goka, H. Matsumoto, and H. Koshiishi, "A new international geostationary electron model: IGE-2006, from $1 \mathrm{keV}$ to 5.2 MeV," Space Weather, vol. 6, 2008.

[24] S. Bourdarie, D. Lazaro, A. Hands, K. Ryden, P. Nieminen, "Electron environment specification models for navigation orbits," Radiation and Its Effects on Components and Systems (RADECS), 2009 European Conference on , pp.364-368, 14-18 Sept. 2009.

[25] J.F. Fennell, H.C. Koons, M.W. Chen, J.B. Blake, "Internal charging: a preliminary environmental specification for satellites," IEEE Trans. Plasma Sci., vol. 28, pp.2029-2036, Dec 2000

[26] T.P. O’Brien, J. F. Fennell, J. L. Roeder, and G. D. Reeves, "Extreme electron fluxes in the outer zone,” Space Weather, 5, S01001, 2007

[27] S. Bourdarie, et al., "Radiation belt representation of the energetic electron environment: Model and data synthesis using the Salammbo radiation belt transport code and Los Alamos geosynchronous and GPS energetic particle data," Space Weather, 3, S04S01, 2005

[28] B. Ni et al., "Reanalyses of the radiation belt electron phase space density using nearly equatorial CRRES and polar-orbiting Akebono satellite observations," J. Geophys. Res., 114, A05208, 2009.

[29] G.D. Reeves, Y. Chen, G. S. Cunningham, R. W. H. Friedel, M. G. Henderson, V. K. Jordanova, J. Koller, S. K. Morley, M. F. Thomsen, and S. Zaharia, "Dynamic Radiation Environment Assimilation Model: DREAM,” Space Weather, 10, S03006, 2012.

[30] V. Maget, S. Bourdarie, D. Boscher, and R. H. W. Friedel, "Data assimilation of LANL satellite data into the Salammbo electron code over a complete solar cycle by direct insertion," Space Weather, 5, S10003, 2007.

[31] V. Maget, S. Bourdarie, D. Boscher, "Direct Data Assimilation Over Solar Cycle Time-Scales to Improve Proton Radiation Belt Models," IEEE Trans. Nucl. Sci., vol.55, no.4, pp.2188-2196, Aug. 2008.

[32] S. Bourdarie, A. Sicard-Piet, R. Friedel, T.P. O'Brien, T. Cayton, B. Blake, D. Boscher, D. Lazaro, "Outer Electron Belt Specification Model," IEEE Trans. Nucl. Sci., vol.56, no.4, pp.2251-2257, Aug. 2009.

[33] G.P. Ginet et al., "AE9/AP9/SPM: Models for specifying the trapped energetic particle and space plasma environment," manuscript in preparation for Space Sci. Rev., unpublished.

[34] T.P. O'Brien, "A framework for next-generation radiation belt models," Space Weather, 3, S07B02, 2005. 
[35] T.P. O’Brien and T.B. Guild, “Trapped Electron Model 2 (TEM-2)”, TR-201(3905)-2, The Aerospace Corporation, El Segundo, CA 2010.

[36] Mewaldt, R.A., "Elemental Composition and Energy of Galactic Cosmic Rays," Proceedings of Workshop on the Interplanetary Charged Particle Environment, Jet Propulsion Laboratory, Pasadena, Ca 121-132 (1988)

[37] Fermi, E., "On the Origin of the Cosmic Radiation," Phys. Rev., 75, 1169-1174 (1949) [Gl79] Gloeckler, G. "Composition of Energetic Particle Populations in Interplanetary Space," Rev Geophys. Space Phys., 17, 569, 1979.

[38] Simpson, J. A. (2000). "The cosmic ray nucleonic component: The invention and scientific uses of the neutron monitor". Space Science Reviews 93

[39] Gombosi, T.I., Physics of the Space Environment, Cambridge University Press, 1998.

[40] Davis, A.J. et al, "Solar Minimum Spectra of Galactic Cosmic Rays and Their Implications for Models of the Near-Earth Environment," J. Geophys. Res., 106, 29,979-29,987, Dec. 2001.

[41] Engelmann, J.J. et al, "Charge Composition and Energy Spectra of Cosmic-Ray Nuclei for Elements from Be to Ni. Results from HEAO-3C2, " Astron. Astrophys., 233, 96-111 (1990)

[42] Juliusson, Einar "Charge Composition and Energy Spectra of Cosmicray Nuclei at Energies above $20 \mathrm{GeV}$ per Nucleon," Astrophys. J., 191, 331-348 (July 1974)

[43] Orth, Charles D., et al, "Abundances and Spectra for Cosmic-ray Nuclei from Lithium to Iron for 2 to $150 \mathrm{GeV}$ Per Nucleon," Astrophys. J., 226, 1147-1161 (December 1978)

[44] Adams, J.H., R. Silberberg, and C.H.Tsao, Cosmic Ray Effects on Microelectronics, Part I: The Near-Earth Particle Environment, NRL Memorandum Report 4506, Naval Research Laboratory, Washington DC, Dec. 1981.

[45] J.H. Adams, Jr., Cosmic Ray Effects on Microelectronics, Part IV, NRL Memorandum Report 5901, Naval Research Laboratory, Washington DC, Dec. 1987.

[46] Nymmik, R.A., "Time Lag of galactic Cosmic Ray Modulation: Conformity to General Regularities and Influence on Particle Energy Spectra," Adv Space Res, 26, No. 11, 1875-2000 (2000)

[47] Tylka, A. J. et al., "CREME96: A Revision of the Cosmic ray Effects on Micro-Electronics Code," IEEE Trans. on Nucl. Sci., 44, 2150 (1997)

[48] Fisk, L.A., Kozlovsky, B. Ramaty, R. "An Interpretation of the Observed Oxygen and Nitrogen Enhancements in Low Energy Cosmic rays," Astrophys. J., Lett., 190, L35-L37, May 1974

[49] Badhwar, G. D. and P.M. O'Neill, "Galactic Cosmic Radiation Model and Its Applications," Advances in Space Research, 17 No. 2, (1996)

[50] P. M. O’Neill, “Badhwar - O’Neill Galactic Cosmic Ray Model Update Based on Advanced Composition Explorer (ACE) Energy Spectra from 1997 to Present,” Advances in Space Research, 37, 1727 (2006)

[51] P. M. O’Neill, "Badhwar - O’Neill Galactic Cosmic Ray Flux Model Revised," Transactions on Nuclear science, 57 (Dec 2010)

[52] Pesses, M.E., J.R. Jokipii, and D. Eichler, "Cosmic ray drift, Shock acceleration, and the Anomalous Component of Cosmic rays," Astrophys. J., 246, L85-L88 (June 1981)

[53] Cliver, E.W. and A.G. Ling, "Lagging behind the solar cycle," Astrophys. J. Lett., 551, L189, 2001

[54] Fujii, Z. and F.B. McDonald, "Radial intensity gradients of galactic cosmic rays (1972-1995) in the heliosphere," J. Geophys. Res., 102, 201-208, 1997

[55] D.V. Reames, "Particle Acceleration at the Sun and in the Heliosphere", Space Sci. Rev., Vol. 90, 413-491 (1999).

[56] M.A. Xapsos, C. Stauffer, J.L. Barth and E.A. Burke, "Solar Particle Events and Self-Organized Criticality: Are Deterministic Predictions of Events Possible?”, IEEE Trans. Nucl. Sci., Vol. 53, 1839-1843 (Aug. 2006).

[57] G. Boffeta, V. Carbone, P. Giuliani, P. Veltri and A. Vulpiani, "Power Laws in Solar Flares: Self-Organized Criticality or Turbulence?” Phys. Rev. Lett., Vol. 83, 4662-4665 (1999).

[58] S.B. Gabriel and G.J. Patrick, "Solar Energetic Particle Events: Phenomenology and Prediction”, Space Sci. Rev., Vol. 107, 55-62 (2003).

[59] R.A. Nymmik, “Improved Environment Radiation Models”, Adv. Space Res., Vol. 40, 313-320 (2007).

[60] J.H. King, "Solar Proton Fluences for 1977-1983 Space Missions", J. Spacecraft, Vol. 11, 401-408 (1974).
[61] J. Feynman, T.P. Armstrong, L. Dao-Gibner and S.M. Silverman, "New Interplanetary Proton Fluence Model”, J. Spacecraft, Vol. 27, 403-410 (1990).

[62] M.A. Xapsos, G. P. Summers, J.L. Barth, E.G. Stassinopoulos and E.A. Burke, "Probability Model for Worst Case Solar Proton Event Fluences”, IEEE Trans. Nucl. Sci., Vol. 46, 1481-1485 (1999).

[63] J. Feynman, G. Spitale, J. Wang and S. Gabriel, "Interplanetary Fluence Model: JPL 1991”, J. Geophys. Res., Vol. 98, 13281-13294 (1993).

[64] E. G. Stassinopoulos and J.H. King, "Empirical Solar Proton Models for Orbiting Spacecraft Applications”, IEEE Trans. Aerospace and Elect. Sys., Vol. 10, 442-450 (1974).

[65] R.A. Nymmik, "Probabilistic Model for Fluences and Peak Fluxes of Solar Energetic Particles”, Radiation Mesurements, Vol.30, 287-296 (1999).

[66] M.A. Xapsos, G.P. Summers, J.L. Barth, E.G. Stassinopoulos and E.A. Burke, "Probability Model for Cumulative Solar Proton Event Fluences”, IEEE Trans. Nucl. Sci., Vol. 47, No. 3, 486-490 (June 2000).

[67] P.T.A. Jiggens et al., "ESA SEPEM Project: Peak Flux and Fluence Model”, IEEE Trans. Nucl. Sci., Vol. 59, No. 4, 1066-1077 (Aug. 2012).

[68] I. Jun, R.T. Swimm, A. Ruzmaikin, J. Feynman, A.J. Tylka and W.F. Dietrich, "Statistics of Solar Particle Events: Fluences, Durations and Time Intervals”, Adv. Space Res., Vol. 40, 304-312 (2007).

[69] B. Taylor, G. Vacanti, E. Maddox and C.I. Underwood, "The Interplanetary Electron Model (IEM)”, IEEE Trans. Nucl. Sci., Vol. 58, 2785-2792 (Dec. 2011).

[70] A.J. Tylka et al., "CREME96: A Revision of the Cosmic Ray Effects on Microelectronics Code”, IEEE Trans. Nucl. Sci., Vol. 44, 2150-2160 (1997).

[71] B.J. Anderson and R.E. Smith, Natural Orbital Environment Guidelines for Use in Aerospace Vehicle Development, NASA Technical Memorandum 4527, Marshall Space Flight Center, Alabama, June 1994.

[72] J.W. Wilson, F.A. Cucinotta, J.L. Shinn, L.C. Simonsen, R.R. Dubbed, W.R. Jordan, T.D. Jones, C.K. Chang and M.Y. Kim, "Shielding from Solar Particle Event Exposures in Deep Space”, Radiat. Meas., Vol. 30, 361-382 (1999).

[73] E.G. Stassinopoulos, G.J. Brucker, D.W. Nakamura, C.A. Stauffer, G.B. Gee and J.L. Barth, "Solar Flare Proton Evaluation at Geostationary Orbits for Engineering Applications”, IEEE Trans. Nucl. Sci., Vol. 43, 369-382 (April 1996).

[74] R.A. Nymmik, “Models Describing Solar Cosmic Ray Events”, Radiat. Meas., Vol. 26, 417-420 (1996).

[75] E. Gumbel, Statistics of Extremes, Columbia University Press, NY, 1958.

[76] A.H-S. Ang and W.H. Tang, Probability Concepts in Engineering Planning and Design, Vol. II, Wiley, NY, 1985.

[77] E. Castillo, Extreme Value Theory in Engineering, Academic Press, Boston, 1988.

[78] P.J. Vail, E.A. Burke and J.P. Raymond, "Scaling of Gamma Dose Rate Upset Threshold in High Density Memories”, IEEE Trans. Nucl. Sci., Vol. 30, 4240-4245 (1983).

[79] P.J. Vail and E.A. Burke, "Fundamental Limits Imposed by Gamma Dose Fluctuations in Scaled MOS Gate Insulators", IEEE Trans. Nucl. Sci., Vol. 31, 1411-1416 (1984).

[80] M.A. Xapsos, "Hard Error Dose Distributions of Gate Oxide Arrays in the Laboratory and Space Environments", IEEE Trans. Nucl. Sci., Vol. 43, 3139-3144 (1996).

[81] E.A. Burke, G.E. Bender, J.K. Pimbley, G.P. Summers, C.J. Dale, M.A. Xapsos and P.W. Marshall, "Gamma Induced Dose Fluctuations in a Charge Injection Device”, IEEE Trans. Nucl. Sci., Vol. 35, 1302-1306 (1988).

[82] P.W. Marshall, C.J. Dale, E.A. Burke, G.P. Summers and G.E. Bender, "Displacement Damage Extremes in Silicon Depletion Regions", IEEE Trans. Nucl. Sci., Vol. 36, 1831-1839 (1989).

[83] P.J. McNulty, L.Z. Scheick, D.R. Roth, M.G. Davis and M.R.S. Tortora, "First Failure Predictions for EPROMs of the Type Flown on the MPTB Satellite”, IEEE Trans. Nucl. Sci., Vol. 47, 2237-2243 (2000).

[84] J.H. Adams, Jr., W.F. Dietrich and M.A. Xapsos, "Probabilistic Solar Energetic Particle Models", Proceedings of the $32^{\text {nd }}$ International Cosmic Ray Conference, Beijing (2011). 
[85] M.A. Xapsos, G.P. Summers and E.A. Burke, "Probability Model for Peak Fluxes of Solar Proton Events”, IEEE Trans. Nucl. Sci., Vol. 45, 2948-2953 (1998).

[86] K.G. McCracken, G.A.M. Dreschoff, E.J. Zeller, D.F. Smart and M.A. Shea, "Solar Cosmic Ray Events for the Period 1561 - 19941. Identification in Polar Ice”, J. Geophys. Res., Vol. 106, 21585-21598 (2001).

[87] E.W. Wolff et al., "The Carrington Event Not Observed in Most Ice Core Nitrate Records”, Geophys. Res. Lett., Vol. 39, L08503 (April 2012).

[88] M.A. Xapsos, C.A. Stauffer, T.M. Jordan, J.H. Adams, Jr. and W.F. Dietrich, "Periods of high intensity solar proton flux", IEEE Trans. Nucl. Sci., Vol. 59, No. 4, 1054-1059 (Aug. 2012).

[89] M.A. Xapsos, C. Stauffer, T. Jordan, J.L. Barth and R. Mewaldt, "Model for Cumulative Solar Heavy Ion Energy and Linear Energy Transfer Spectra”, IEEE Trans. Nucl. Sci., Vol. 54, 1985-1989 (Dec. 2007).

[90] D.L. Chenette, J. Chen, E. Clayton, T.G. Guzik, J.P. Wefel, M. GarciaMunoz, C. Lopate, K.R. Pyle, K.P. Ray, E.G. Mullen and D.A. Hardy, "The CRRES/SPACERAD Heavy Ion Model of the Environment (CHIME) for Cosmic Ray and Solar Particle Effects on Electronic and Biological Systems in Space”, IEEE Trans. Nucl. Sci., Vol. 41, 23322339 (1994).

[91] P.L. McKerracher, J.D. Kinnison and R.H. Maurer, "Applying New Solar Particle Event Models to Interplanetary Satellite Programs”, IEEE Trans. Nucl. Sci., Vol. 41, 2368-2375 (1994).

[92] P.P. Majewski, E. Normand and D.L. Oberg, “A New Solar Flare Heavy Ion Model and its Implementation through MACREE, an Improved Modeling Tool to Calculate Single Event Effect Rates in Space”, IEEE Trans. Nucl. Sci., Vol. 42, 2043-2050 (1995).

[93] A.J. Tylka, W.F. Dietrich, P.R. Boberg, E.C. Smith and J.H. Adams, Jr., "Single Event Upsets Caused by Solar Energetic Heavy Ions", IEEE Trans. Nucl. Sci., Vol. 43, 2758-2766 (1996).

[94] C.S. Dyer, K. Hunter, S. Clucas, D. Rodgers, A. Campbell and S. Buchner, "Observation of Solar Particle Events from CREDO and MPTB During the Current Solar Maximum”, IEEE Trans. Nucl. Sci., Vol. 49, 2771-2775 (2002).

[95] J.S. Neal and L.W. Townsend, "Predicting Dose-Time Profiles of Solar Energetic Particle EventsUsing Bayesian Forecasting Methods”, IEEE Trans. Nucl. Sci., Vol. 48, 2004-2009 (2001). 


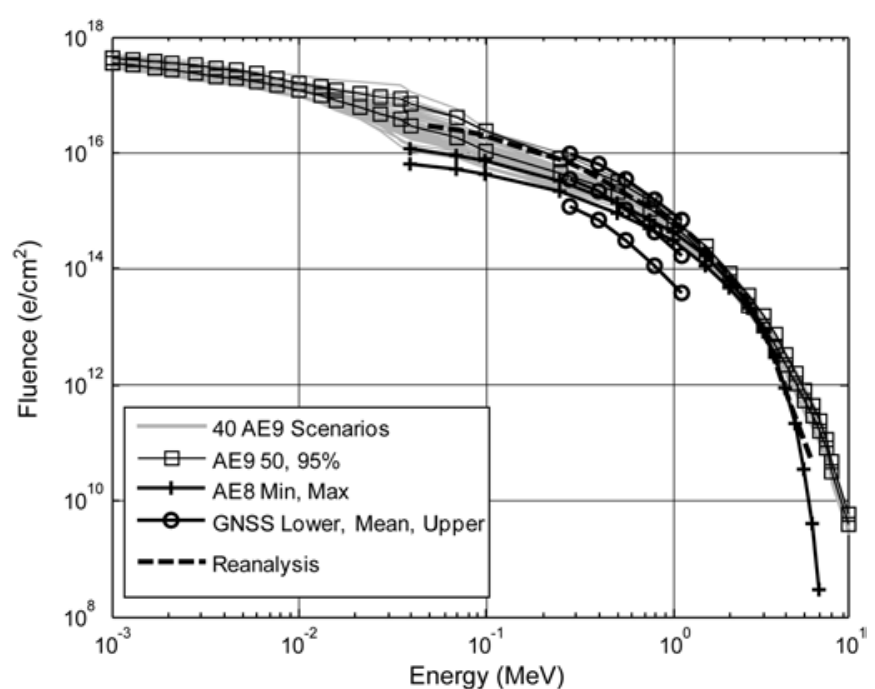

Fig. 1. Several specifications of the 10-year electron fluence at a GPS orbit.

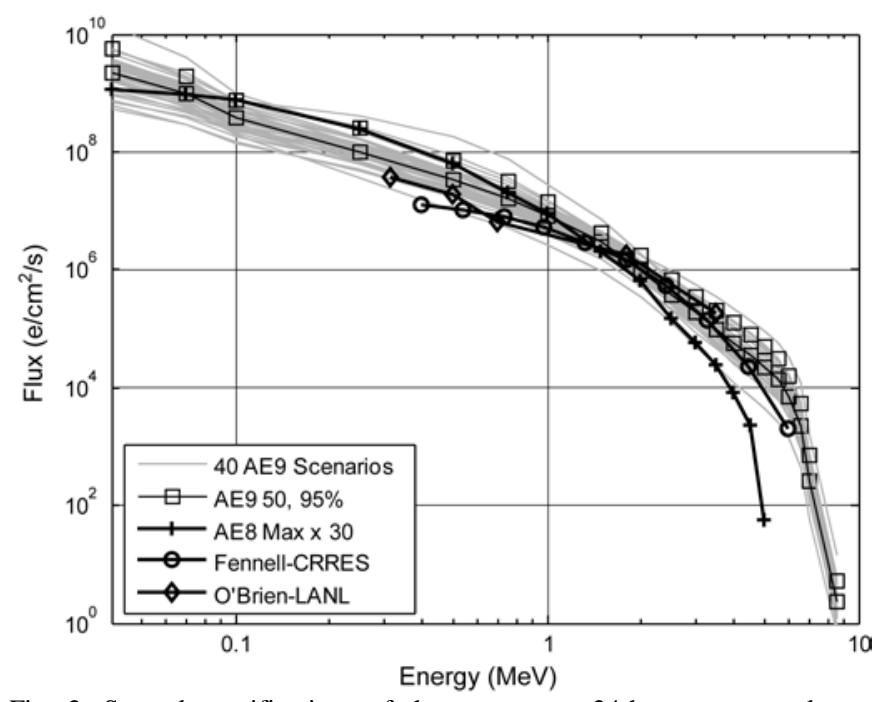

Fig. 2. Several specifications of the worst case 24-hour average electron environment at geostationary orbit for internal charging.

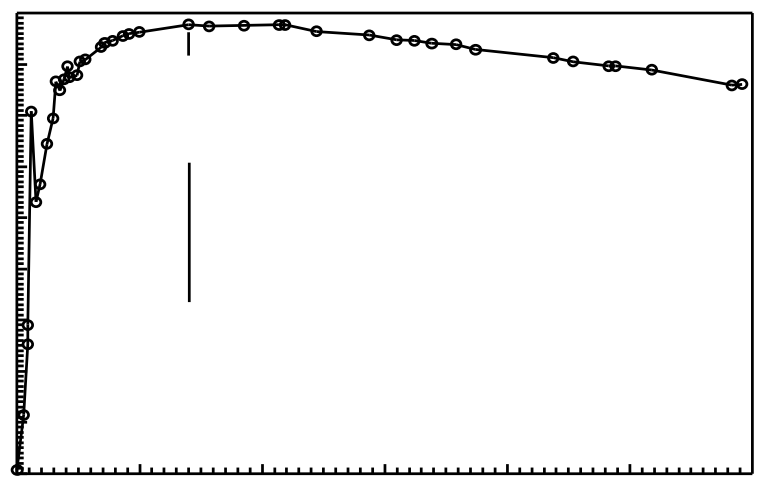

Fig. 3. Since iron has the highest binding energy of all the elements, heat is produced by combining lighter nuclei (fusion) and by splitting nuclei for elements heavier than iron (fission).

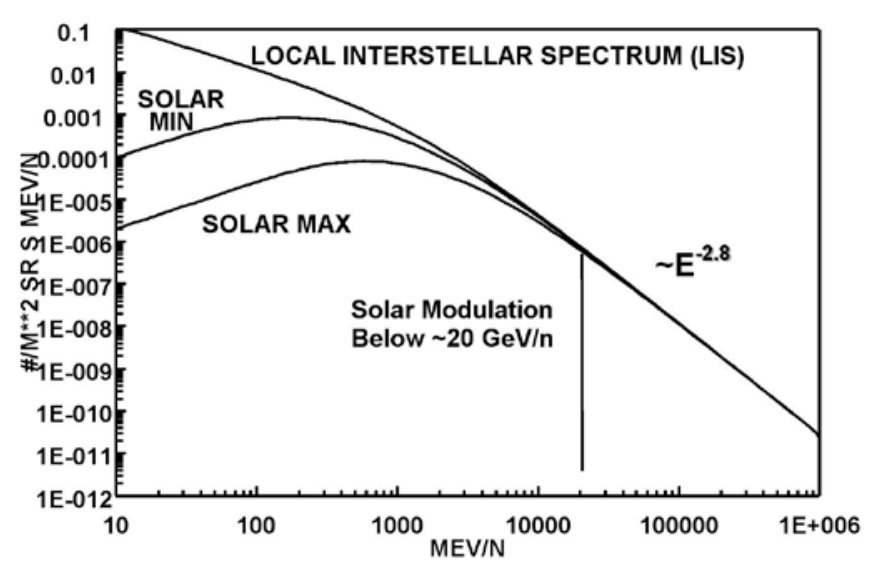

Fig. 4. Differential energy spectra for a typical GCR element - iron

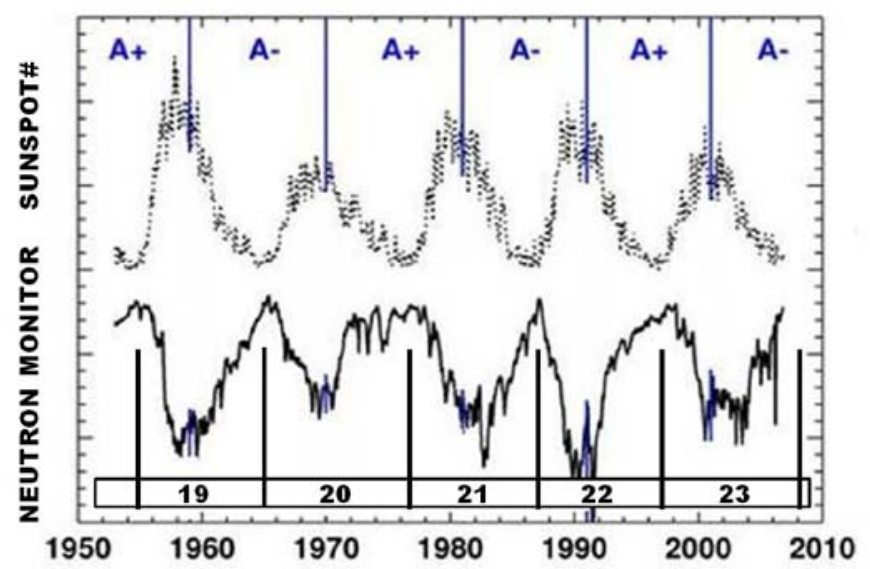

Fig. 5. Neutron monitor, sunspot count, solar magnetic field, and cycle. Neutron monitor anti-correlates with sunspot numbers and shows an 11-year cycle with "pointed" and "plateau" shaped peak intensity occurring on alternate cycles that the sunspot number does not exhibit.

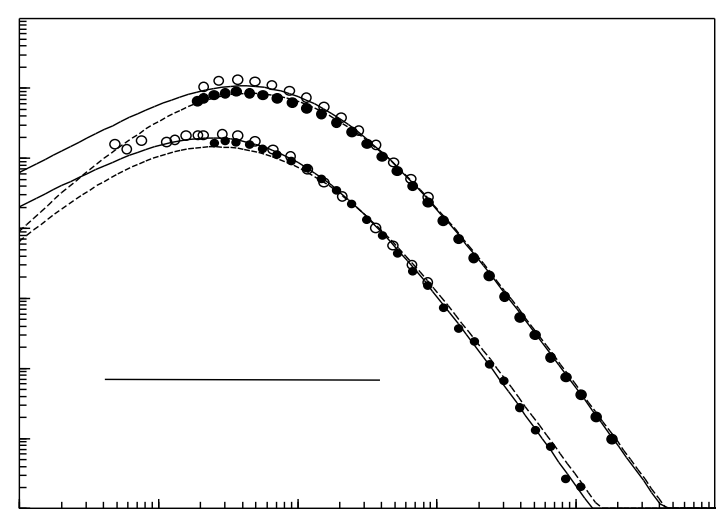

Fig. 6. BESS and IMAX provide the best available proton and helium spectra measurements. 


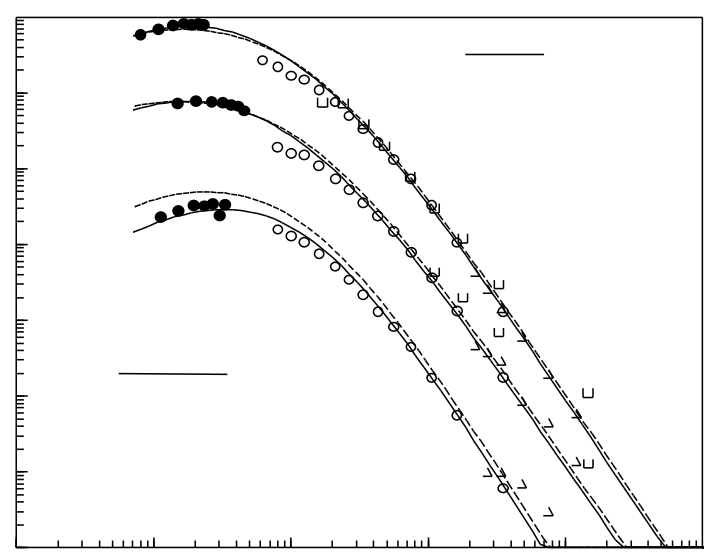

Fig. 7 Low energy (ACE) and High energy (HEAO, JUL [42] and ORTH [43]) spectra for Oxygen, Iron, and Phosphorus. These data are typical of that available for all the elements from $\operatorname{Li}(\mathrm{Z}=3)$ to $\mathrm{Ni}(\mathrm{Z}=28)$. Phosphorus is a scarce element and little (if any) data were available for it to Nymmik in 1997.

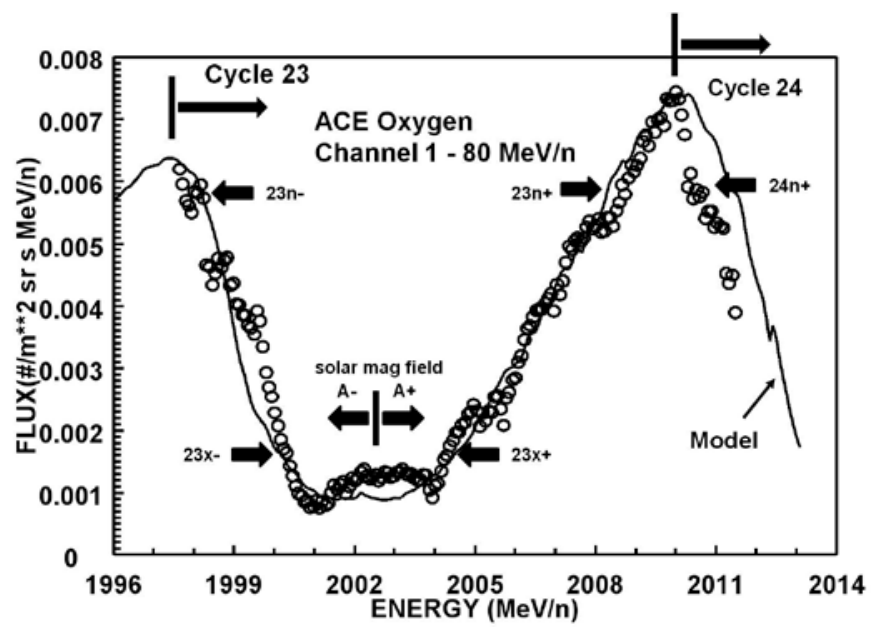

Fig. 8. ACE CRIS (channel $1 \sim 80 \mathrm{MeV} / \mathrm{n}$ ) oxygen flux for solar cycles 23 and part of 24. The symbols (eg 23n-, 23n+, and 24n+) correspond to Fig. 9 and have the same solar modulation at different solar magnetic field (field changes sign near solar maximum) and cycle conditions.

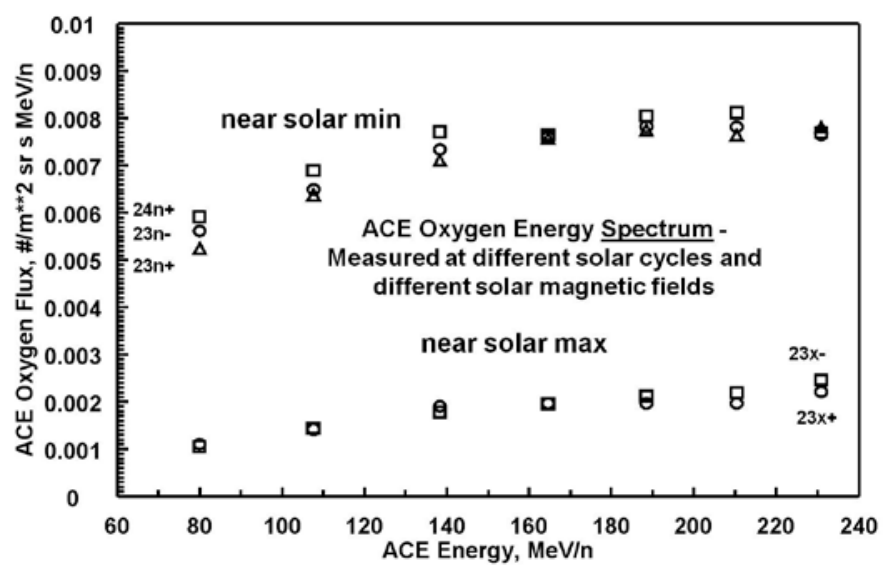

Fig. 9. ACE CRIS (channel 1 - 7) oxygen flux for different solar field and cycle conditions - but for $\sim$ the same solar modulation. The symbols (eg $23 n-$, $23 n+, 24 n+$ ) correspond to Fig. 8 and indicate the solar conditions at the time selected.

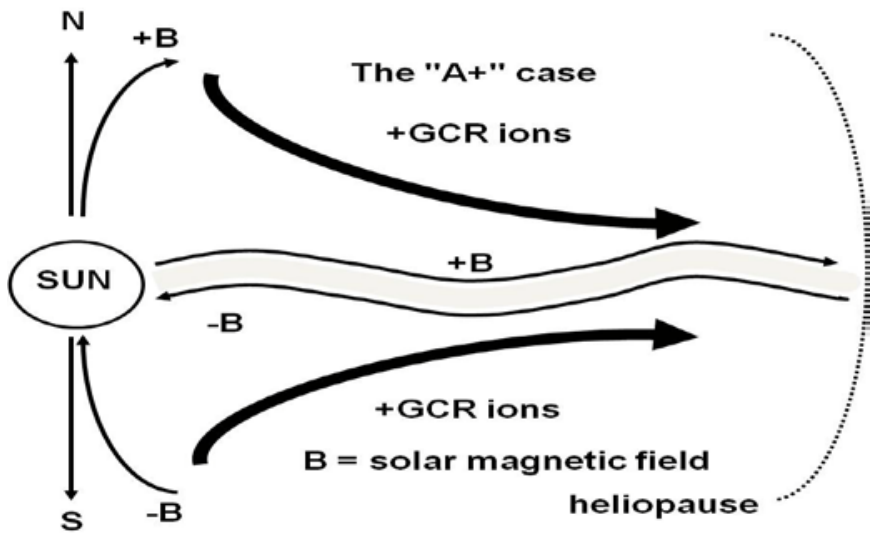

Fig. 10. Gradient and curvature drift allows positive GCR ions to enter the heliosphere from the polar regions (both North and South) and exit along the neutral current sheet (where $\mathrm{B}=0$ ) when the solar magnetic field points outward in the northern hemisphere.

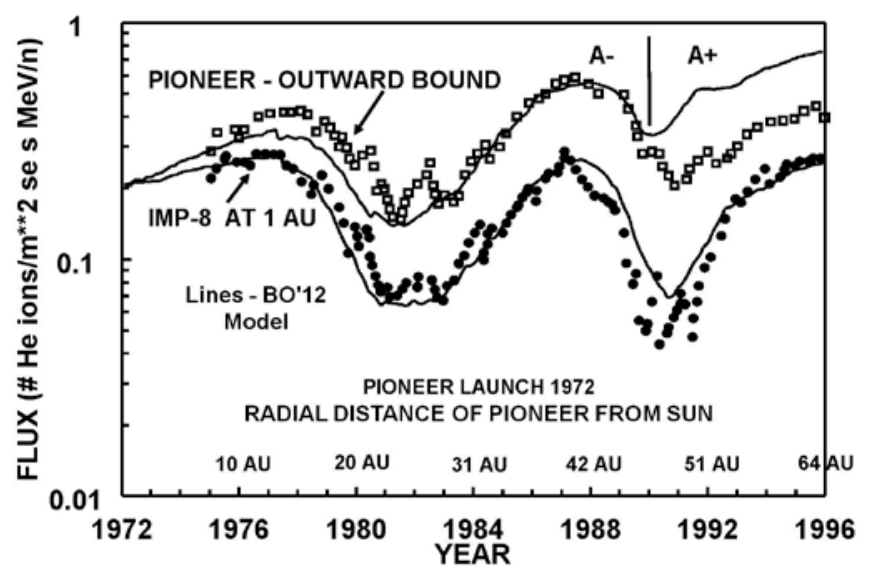

Fig. 11. Pioneer 10 helium flux at $\sim 300 \mathrm{MeV} / \mathrm{n}$ as the spacecraft progressed out into the deep heliosphere. IMP-8 (at $1 \mathrm{AU}$ ) helium flux at the same energy is also shown. Model matches Pioneer well until field reversal, A- to A+.

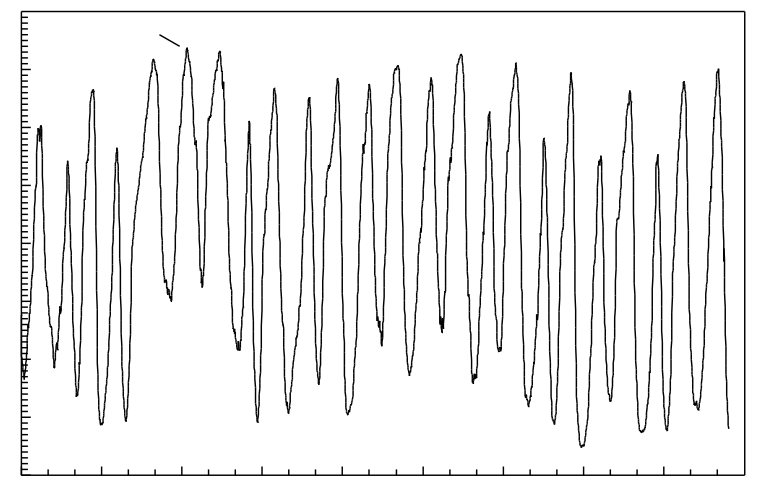

Fig. 12. Flux of $80 \mathrm{MeV} / \mathrm{n}$ GCR oxygen derived from the sunspot number using BO model for cycles1 to 24. 


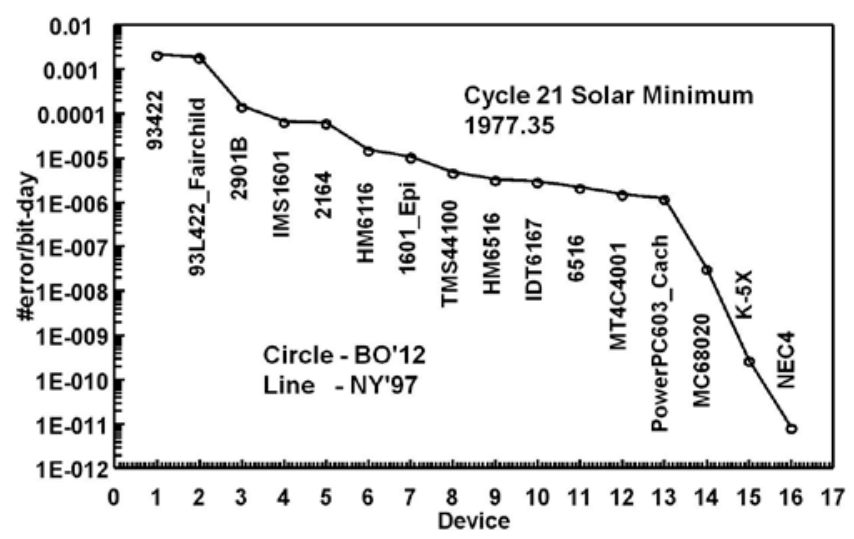

Fig. 13. SEE error rate for BO and Nymmik GCR models for typical microelectronic components in deep space (100 mil aluminum) at solar minimum.

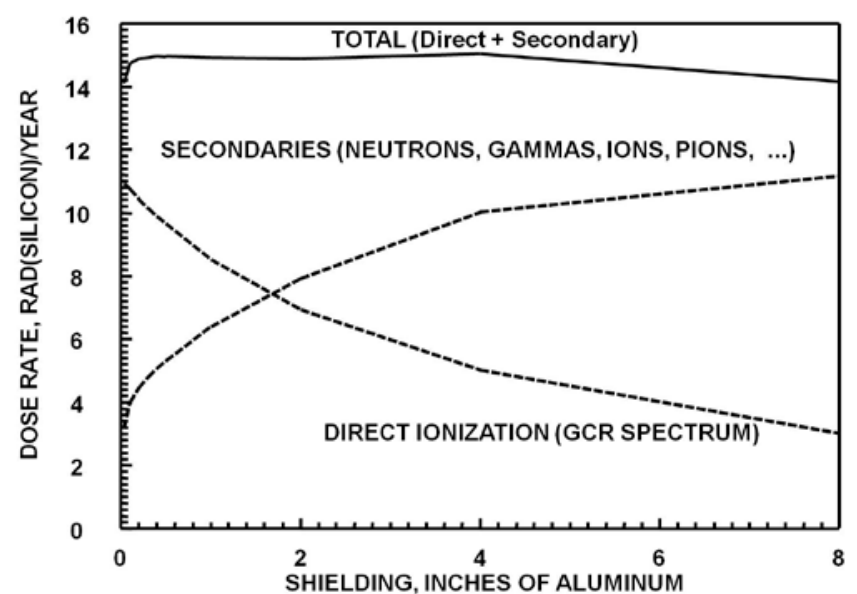

Fig. 14. Annual dose for GCRs for Cycle 21 Solar Minimum (1977.35) calculated by the FLUKA nuclear code.

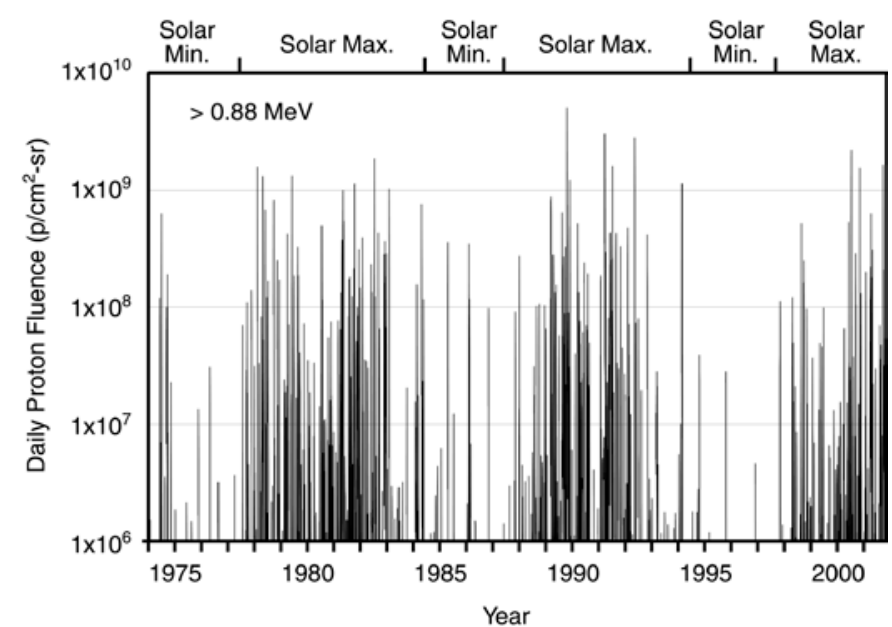

Fig. 15. Daily fluences of $>0.88 \mathrm{MeV}$ protons due to solar particle events between approximately 1974 and 2002.

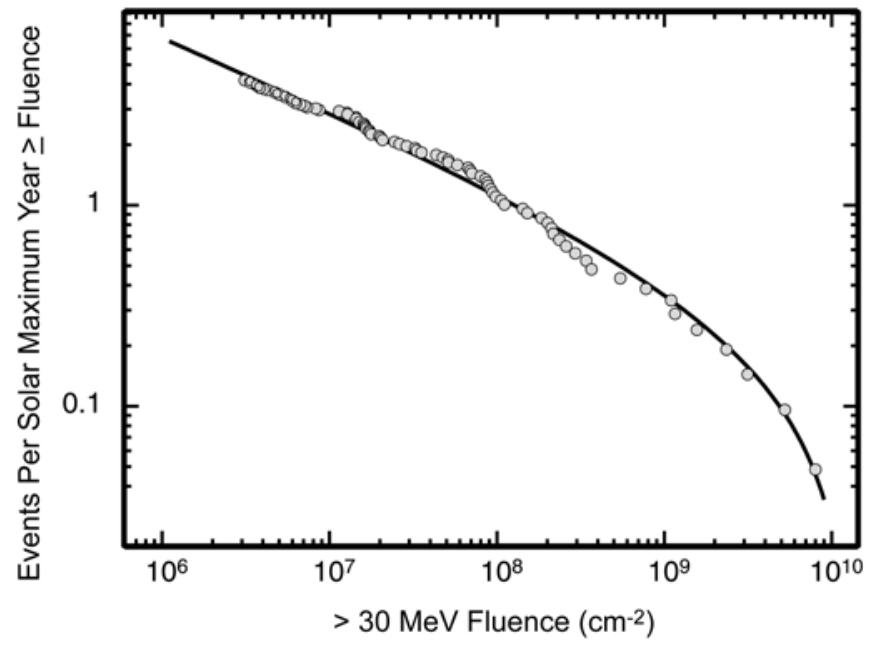

Fig. 16. Comparison of the truncated power law distribution to 3 solar cycles of data during solar maximum [62].

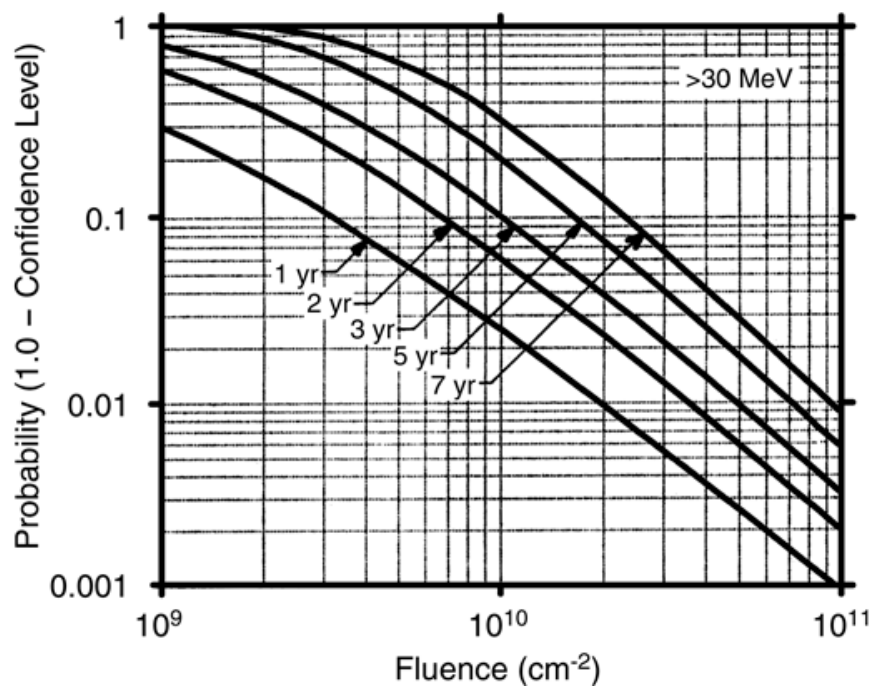

Fig. 17. JPL91 solar proton fluence model for $>30 \mathrm{MeV}$ protons. The misprint of $\mathrm{x}$-axis units has been corrected from the original reference [61].

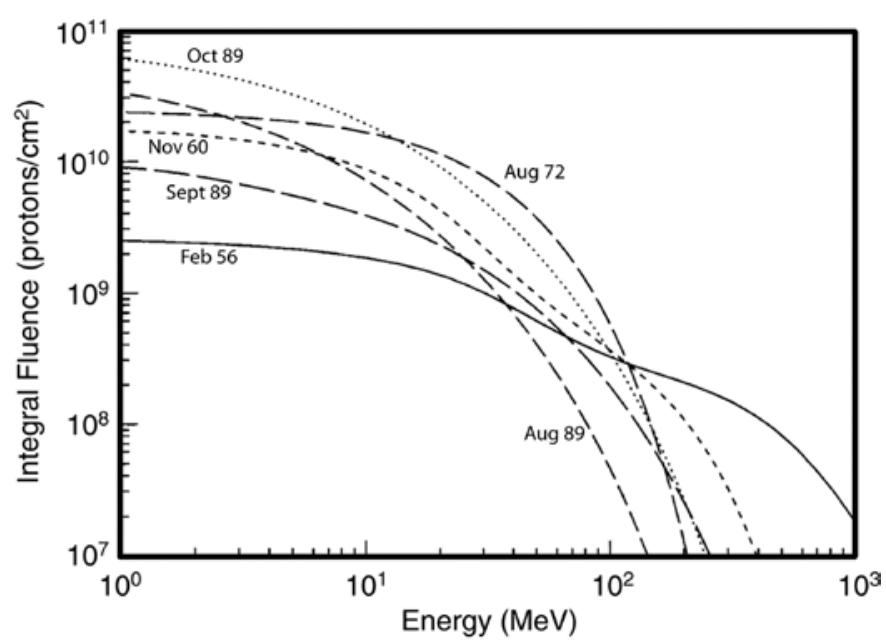

Fig. 18. Some of the most severe solar proton event energy spectra in solar cycles 19-22 [72]. Curves are labeled with the event month and year. 


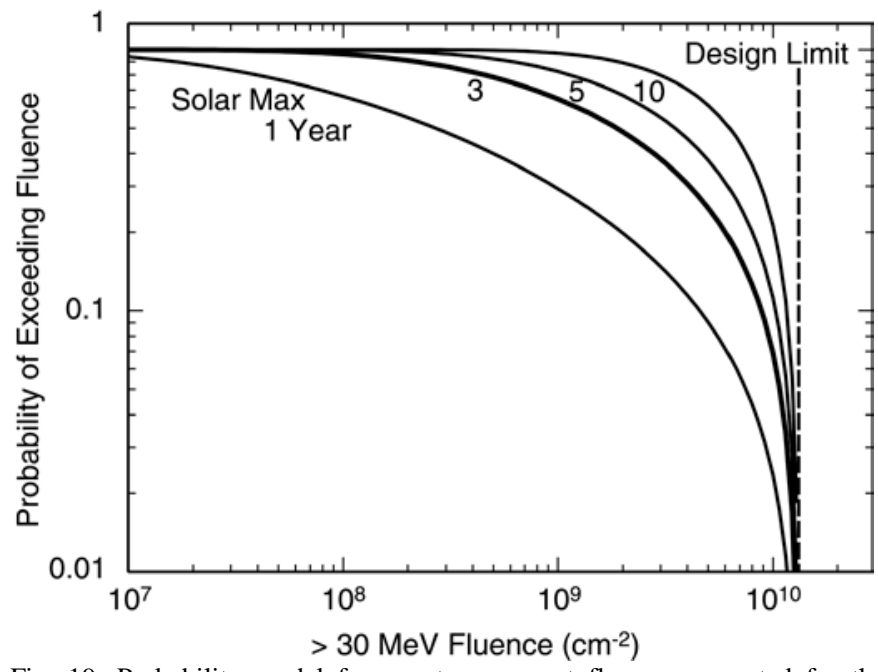

Fig. 19. Probability model for worst-case event fluences expected for the indicated time periods during solar maximum [62].

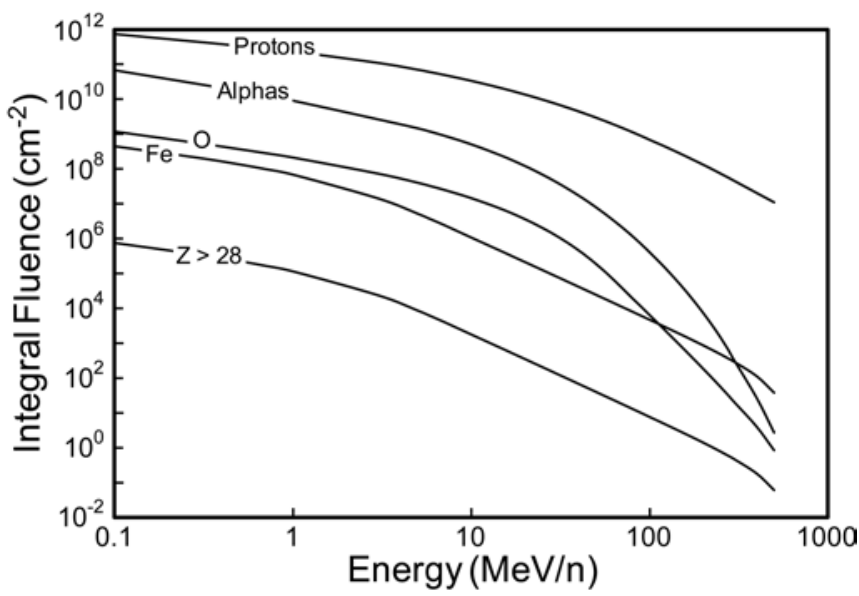

Fig. 20. Integral fluence-energy spectra for protons, alpha particles, oxygen, iron and summed spectra for $\mathrm{Z}>28$ elements for a 1-year mission during solar maximum at the $95 \%$ confidence level. Calculations are done with the ESP/PSYCHIC model [89].

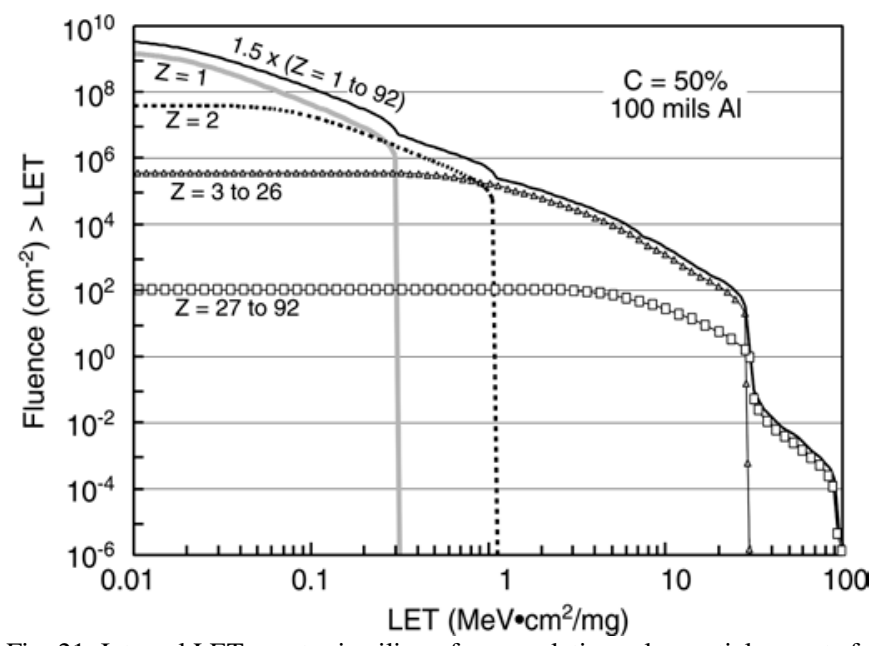

Fig. 21. Integral LET spectra in silicon for cumulative solar particle events for a 2-year mission during solar maximum at the $50 \%$ confidence level. The spectra illustrate the contributions of $\mathrm{Z}=1$ (gray line), $\mathrm{Z}=2$ (dashed line), $\mathrm{Z}$ $=3$ to 26 (triangles), and $Z=27$ to 92 (squares) ions to the total spectrum (solid black line). The total has been multiplied by a factor of 1.5 for clarity [89].

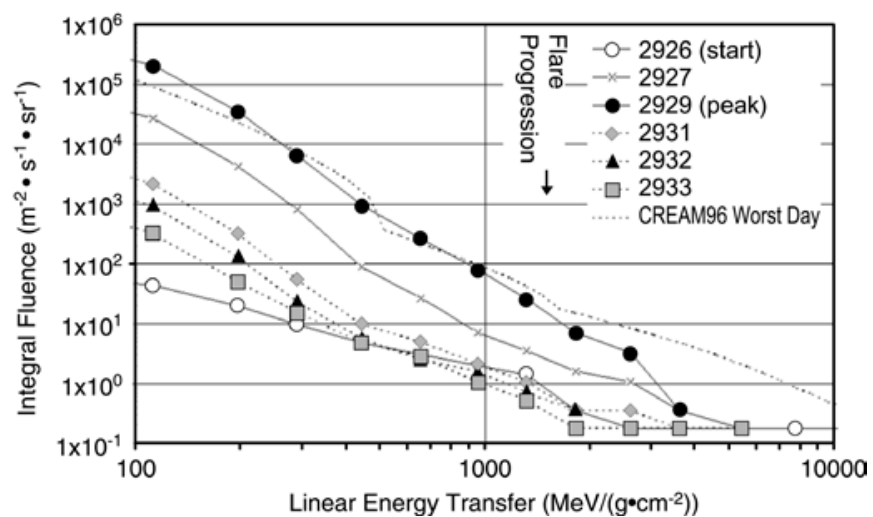

Fig. 22. Comparison of a solar heavy ion event that occurred in November 2001 with the CREME96 "worst day" model. The progression of daily intensities is indicated with the peak intensity occurring on day 2929 of the mission [94]. 
$>$ REPLACE THIS LINE WITH YOUR PAPER IDENTIFICATION NUMBER (DOUBLE-CLICK HERE TO EDIT) <

TABLE I

PARTICLE RADIATIONS IN NEAR-EARTH ORBIT AND SOME PROPERTIES

\begin{tabular}{|c|c|c|c|c|}
\hline Radiation & $\begin{array}{l}\text { Maximum } \\
\text { Energy }\end{array}$ & $\begin{array}{l}\text { Maximum } \\
\text { Flux }\end{array}$ & $\begin{array}{l}\text { Radiation } \\
\text { Effects }\end{array}$ & $\begin{array}{c}\text { Shielding } \\
\text { Effectiveness }\end{array}$ \\
\hline $\begin{array}{l}\text { Trapped } \\
\text { Protons }\end{array}$ & $\sim 500 \mathrm{MeV}$ & $\begin{array}{l}\sim 10^{5} \mathrm{~cm}^{-2} \mathrm{~s}^{-1} \\
(>10 \mathrm{MeV})\end{array}$ & TID, DD, SEE & Moderate \\
\hline $\begin{array}{l}\text { Trapped } \\
\text { Electrons }\end{array}$ & $\sim 10 \mathrm{MeV}$ & $\begin{array}{l}\sim 10^{6} \mathrm{~cm}^{-2} \mathrm{~s}^{-1} \\
(>1 \mathrm{MeV})^{\mathrm{a}}\end{array}$ & TID, DD, ESD & High \\
\hline $\begin{array}{l}\text { Glactic } \\
\text { Cosmic Rays }\end{array}$ & $\sim 10^{11} \mathrm{GeV}$ & $\sim 10 \mathrm{~cm}^{-2} \mathrm{~s}^{-1}$ & SEE & Low \\
\hline $\begin{array}{l}\text { Solar Particle } \\
\text { Events }\end{array}$ & $\sim 10 \mathrm{GeV} / \mathrm{n}$ & $\begin{array}{c}\sim 10^{5} \mathrm{~cm}^{-2} \mathrm{~s}^{-1} \\
(>10 \mathrm{MeV} / \mathrm{n})\end{array}$ & TID, DD, SEE & Moderate \\
\hline
\end{tabular}

${ }^{\bar{a}}$ Quiet time conditions
TABLE II

TRAPPED RADIATION MODEL REQUIREMENTS ${ }^{\mathrm{a}}$

\begin{tabular}{llll}
\hline \hline Priority & PopUlation & \multicolumn{1}{c}{ Energy } & \multicolumn{1}{c}{ Location } \\
\hline 1 & Protons & $\begin{array}{l}>10 \mathrm{MeV} \\
(>80 \mathrm{MeV})\end{array}$ & LEO, MEO \\
& & $>1 \mathrm{MeV}$ & LEO, MEO, GEO \\
2 & Electrons & $30 \mathrm{eV}-100 \mathrm{keV}$ & LEO, MEO, HEO, GEO \\
3 & Plasma & $(30 \mathrm{eV}-5 \mathrm{keV})$ & \\
& & $100 \mathrm{keV}-1 \mathrm{MeV}$ & MEO, GEO \\
4 & Electrons & $0.1-10 \mathrm{MeV}$ & LEO, MEO, GEO \\
5 & Protons & $(5-10 \mathrm{MeV})$ & \\
\hline \hline
\end{tabular}

${ }^{\mathrm{a}}$ After Table 2 of [14]. Parentheses indicate higher priority energy ranges. 